\title{
ON SIMPLICITY CONCEPTS FOR ERGODIC ACTIONS
}

\author{
Alexandre I. DAnilenko
}

\begin{abstract}
A weakly mixing measure preserving action of a locally compact second countable group on a standard probability space is called 2-fold near simple if every ergodic joining of it with itself is either product measure or is supported on a 'convex combination' of graphs. A similar definition can be given for near simplicity of higher order. This generalizes Veech-del Junco-Rudolph notion of simplicity. Main results: An analogue of Veech theorem on factors holds for the 2-fold near simple actions. A weakly mixing group extension of an action with near MSJ is near simple. The action of a normal co-compact subgroup is near simple if and only if the whole action is near simple. The subset of all 2 -fold near simple transformations (i.e. $\mathbb{Z}$-actions) is meager in the group of measure preserving transformations endowed with the weak topology. Via the $(C, F)$-construction, we produce a near simple quasi-simple transformation which is disjoint from any simple map.
\end{abstract}

\section{INTRODUCTION}

By a dynamical system we mean a measure preserving action $T$ of a locally compact second countable group $G$ on a standard probability space $(X, \mathfrak{B}, \mu)$. An $l$-fold self-joining of $T$ is a measure on $X^{l}$ which is invariant under the product $G$-action $T \times \cdots \times T$ and whose coordinate marginals are all $\mu$.

The fundamental concepts of simplicity and minimal self-joinings (MSJ) of any order for ergodic dynamical systems were introduced and deeply investigated by A. del Junco and D. Rudolph in [dJR]. (Historically, D. Rudolph introduced a stronger version of MSJ in [Ru] and W. Veech introduced 2-fold simplicity in [Ve] for $\mathbb{Z}$-actions only.) Basically, these properties mean that a dynamical system $T$ has only 'obvious' ergodic self-joinings: product measure and measures supported on graphs. A substantial information about $T$ can be derived from the fact that $T$ is simple, especially about the structure of factors of $T$, the centralizer of $T$, and also on the way $T$ can be joined with other dynamical systems. Moreover, transformations with MSJ is a source of strike counterexamples in ergodic theory $[\mathrm{Ru}]$.

However the concepts of simplicity and MSJ elaborated mainly to investigate $\mathbb{Z}$ and $\mathbb{R}$-actions seem to be somewhat restrictive for non-Abelian dynamical systems. We encountered with that in [Da3] when constructing mixing rank one actions of countable sums of finite groups. Though it is natural to expect-by analogy with the $\mathbb{Z}$-case - that such actions have MSJ, one can see easily that there exist no MSJ-actions of any group containing a non-central element whose conjugacy class

1991 Mathematics Subject Classification. 37A15, 37A05.

Key words and phrases. Joining, simple action, MSJ.

The work was supported in part by CRDF, grant UM1-2546-KH-03. 
is finite. To overcome this 'conflict' we gave in [Da3] another - more generaldefinition of MSJ (called near MSJ in the present paper) and showed that the actions under question have near MSJ. We note that for Abelian $G$, the notions of MSJ and near MSJ are equivalent.

In this paper we introduce a companion (to near MSJ) property of near simplicity which is more general then the simplicity. To this end we just enlarge the list of 'obvious' ergodic 2-fold self-joinings of $T$. Now it consists of product measure and measures supported on convex combinations of graphs. In a similar way we define near simplicity of higher orders. However, in contrast to near MSJ, even the class of near simple $\mathbb{Z}$-actions is wider then the class of simple ones. Our purpose in this paper is to investigate the properties of near simple systems, compare them with simple ones, and produce examples of near simple non-simple transformations.

Some other important generalizations of the simplicity were suggested recently in [RyT] and [dJL2]. A transformation is called 2-fold quasi-simple (2-fold distally simple) if every ergodic non-product 2 -fold self-joining of it is isometric (relatively distal) over the marginals. If, in addition, the system is pairwise independently determined (PID) then it is called QS or DS respectively. Historically, a weaker concept of QS due to V. Ryzhikov and J.-P. Thouvenot appeared in [Ry2]-the forementioned marginal projections were assumed finite-to-one their. (Only minor modifications are needed to extend the results of [Ry2] and [RyT] to the class of DS transformations.) A factor of a simple transformation is QS [dJR]; each non-zero time transformation of a horocycle flow is QS [Th1]. A detailed analysis of infinite self-joinings of DS systems was given in [dJL2] and some disjointness resultsextending those from [dJL1] and [Th2] — were established in [dJL2] and [RyT]. For instance, every weakly mixing 2-fold DS transformation is disjoint in the sense of Furstenberg [Fu] with all so-called well divisible transformation [RyT] (Gaussian transformations are well divisible) and every weakly mixing DS transformation is disjoint with all infinitely divisible maps [dJL2]. However a possible relation between simple and distally simple maps remained unclear. As was shown in [Th1] each non-zero time automorphism of a horocycle flow is a factor of a simple map. In view of that J.-P. Thouvenot asks:

- whether each QS-transformation is a factor of (an isometric extension of) a simple map?

A version of that appears also in [dJL2]:

- whether each DS transformation is a factor of a distal extension of a simple map?

We answer both questions in this paper by constructing a near simple QS transformation which is disjoint from any simple map. Hence it is disjoint from any distal extension of any simple map by [Fu]. It remains to note that disjoint systems do not have common factors.

The paper is organized as follows. Section 1 contains some background material on factors, extensions and joining theory. In Section 2 we introduce the 2-fold near simplicity and near $\mathrm{MSJ}_{2}$ (Definition 2.4) and prove an extension of Veech theorem: every non-trivial factor of a 2-fold near simple action $T$ is the $\sigma$-algebra of subsets fixed by a compact $T$-invariant subgroup $K \subset \operatorname{Aut}_{0}(X, \mu)$, i.e. $T_{g} K T_{g}^{-1}=K$ for all $g \in G$ (Theorem 2.6). In particular, if $T$ is free and has near $\mathrm{MSJ}_{2}$ then we obtain a one-to-one correspondence between the non-trivial factors of $T$ and the compact normal subgroups of $G$ (Corollary 2.7). Notice however that in general different 
compact $T$-invariant subgroups of $\operatorname{Aut}_{0}(X, \mu)$ can determine the same factor of $T$ (Example 2.3).

In Section 3 we define near simplicity and near MSJ of any order (Definition 3.1). We show that given a co-compact normal subgroup $H \subset G$, the $H$-subaction is $l$ fold near simple if and only if so is $T$ (Proposition 3.2). In Proposition 3.4 we give a sufficient condition for a factor of an $l$-fold near simple action to be $l$-fold near simple (see also Proposition 3.6). A weakly mixing compact group extension of an action with near $\mathrm{MSJ}_{l}$ is $l$-fold near simple (Proposition 3.8).

In Section 4, we show that the set of 2-fold near simple transformations is meager in the transformation group $\operatorname{Aut}_{0}(X, \mu)$ endowed with the usual weak topology (Theorem 4.9). This refines a recent result of O. Ageev [Ag2], who showedanswering a question from [dJR] - that the subset of 2 -fold simple transformations is meager. Notice that even in case of simple maps, our proof is different-shorterthan Ageev's one since we bypass the approximation technique which was crucial in his argument. Instead, we apply a couple of well known facts from the cohomology theory of ergodic dynamical systems (Lemma 4.3).

In Section 5, we construct a family of transformations which are near simple but not 2-fold simple (Theorem 5.3). However, all of them have simple factors.

Section 6 contains our main result, an example of near simple QS-transformation $T$ which is disjoint from any simple map (Theorem 6.9). Moreover, $T$ is not prime and it has no prime factors. To construct this transformation we make use of the approach suggested by A. del Junco in $[\mathrm{dJ}]$ which, in turn, is based on original ideas of D. Ornstein [Or]. As in $[\mathrm{dJ}]$, we first construct a funny rank-one action of an auxiliary group $G$-a semidirect product of $\bigoplus_{i=1}^{\infty} \mathbb{Z} / 3 \mathbb{Z}$ with $\mathbb{Z}$ - and then restrict it to $\mathbb{Z}$ (del Junco considered the direct product $\left(\bigoplus_{i=1}^{\infty} \mathbb{Z} / 2 \mathbb{Z}\right) \oplus \mathbb{Z}$ ). Since $G$ is noncommutative, we give - as a byproduct - a partial affirmative answer to a question from $[\mathrm{Ma}]$ : whether the techniques of $[\mathrm{dJ}]$ and $[\mathrm{Ma}]$ extend to non-Abelian actions? Notice that the construction from [dJ], called the $(C, F)$-construction in [Da2], is an algebraic version of the classical geometric cutting-and-stacking. In this paper, we make a step further in its 'algebraization'. For instance, we bypass the nomenclature of blocks and overlaps which is common for [Or], [dJ], [Ma]. When analyzing the joinings of $T$, we apply the pointwise ergodic theorem of E. Lindenstrauss [Li].

In the final Section 7 we state several open problems related to near simple actions.

Acknowledgements. The author is grateful to M. Lemańczyk for stimulating discussions and helpful suggestions. Also I would like to thank V. Ryzhikov for his useful remarks and historical comments. I also thank the referee who found a gap in the original proof of Proposition 2.2.

\section{Preliminaries}

Our purpose in this section is to introduce some notation, remind basic concepts and review a recent progress in the joining theory. We refer to [dJR], [Th1] and [Gl] for a detailed exposition.

Measures invariant under co-compact subactions. Let $G$ be a locally compact second countable group and $(Y, \mathfrak{F})$ a standard Borel $G$-space. Given a cocompact subgroup $H \subset G$, we denote by $\lambda_{H \backslash G}$ normalized Haar measure on the homogeneous right $G$-space $H \backslash G$ and by $s: H \backslash G \rightarrow G$ a Borel cross-section of 
the natural quotient $G \rightarrow H \backslash G$. Then $s(x) g=h(x, g) s(x g)$ for all $x \in H \backslash G$ and $g \in G$, where $h:(H \backslash G) \times G \rightarrow H$ is the corresponding 1-cocycle, i.e. a Borel map such that $h\left(x, g_{1} g_{2}\right)=h\left(x, g_{1}\right) h\left(x g_{1}, g_{2}\right)$ for all $x \in H \backslash G$ and $g_{1}, g_{2} \in G$. Denote by $\mathcal{P}_{G}^{e}$ and $\mathcal{P}_{H}^{e}$ the spaces of ergodic $G$-invariant and ergodic $H$-invariant (resp.) probabilities on $(Y, \mathfrak{F})$. For a measure $\nu \in \mathcal{P}_{H}^{e}$, we let

$$
\widehat{\nu}:=\int_{H \backslash G} \nu \circ s(x) d \lambda_{H \backslash G}(x) .
$$

For completeness, we state with a proof the following 'folklore' result.

Lemma 1.1. The measure $\widehat{\nu}$ is G-invariant and ergodic. The map $\mathcal{P}_{H}^{e} \ni \nu \mapsto \widehat{\nu} \in$ $\mathcal{P}_{G}^{e}$ is onto. Moreover, if $H$ is normal then (1-1) is the H-ergodic decomposition of $\widehat{\nu}$.

Proof. For any $g \in G$, we have

$$
\begin{aligned}
\widehat{\nu} \circ g & =\int_{H \backslash G} \nu \circ(s(x) g) d \lambda_{H \backslash G}(x) \\
& =\int_{H \backslash G}(\nu \circ h(x, g)) \circ s(x g) d \lambda_{H \backslash G}(x) \\
& =\int_{H \backslash G} \nu \circ s(x) d \lambda_{H \backslash G}(x),
\end{aligned}
$$

i.e. $\widehat{\nu}$ is $G$-invariant. Let $A$ be a $G$-invariant subset from $\mathfrak{F}$. Since it is also $H$ invariant, $\nu(A)=0$ or 1 . Hence $\nu \circ s(x)(A)=0$ for all $x \in H \backslash G$. It follows that $\widehat{\nu}(A)=0$ or 1 , i.e. $\widehat{\nu}$ is $G$-ergodic.

Now let $\lambda \in \mathcal{P}_{G}^{e}$. Since $\lambda$ is $H$-invariant, consider its ergodic decomposition $\lambda=\int_{\mathcal{P}_{H}^{e}} \rho d \kappa(\rho)$. Since $\lambda=\int_{H \backslash G} \lambda \circ s(x) d \lambda_{H \backslash G}(x)$, it follows that $\lambda=\int_{\mathcal{P} e} \widehat{\rho} d \kappa(\rho)$. By the first part of the proof, $\widehat{\rho} \in \mathcal{P}_{G}^{e}$. Hence by extremality of $\lambda, \lambda=\widehat{\rho}$ for $\kappa$-a.a. $\rho$.

As for the latter claim in the statement of this lemma, it suffices to note that $\nu \circ g$ is $H$-invariant (and ergodic) for any $g$ if $H$ is normal.

Joinings of ergodic actions. Let $T$ and $S$ be two ergodic $G$-actions on standard probability spaces $(X, \mathfrak{B}, \mu)$ and $\left(Y, \mathfrak{B}_{Y}, \nu\right)$ respectively. We denote by $J(T, S)$ the space of joinings of $T$ and $S$, i.e. the set of all $T \times S$-invariant probabilities on $X \times Y$ whose marginals on $X$ and $Y$ are $\mu$ and $\nu$ respectively. Recall that $T \times S$ stands for the product $G$-action $\left(T_{g} \times S_{g}\right)_{g \in G}$. Following [Fu], we say that $T$ and $S$ are disjoint if $J(T, S)=\{\mu \times \nu\}$. For instance, a weakly mixing $G$-action is disjoint with any $G$-action having a pure point spectrum. In a similar way one can define the set of joinings $J\left(T^{(1)}, \ldots, T^{(l)}\right)$ for any finite (or even countable) family $T^{(1)}, \ldots, T^{(l)}$ of ergodic $G$-actions. The subset of ergodic joinings from $J\left(T^{(1)}, \ldots, T^{(l)}\right)$ is denoted by $J^{e}\left(T^{(1)}, \ldots, T^{(l)}\right)$. If $T^{(1)}=\cdots=T^{(l)}=: T$, we write $J_{l}(T)$ for $J\left(T^{(1)}, \ldots, T^{(l)}\right)$ and call every element of this set an l-fold self-joining of $T$.

Denote by $\operatorname{Aut}_{0}(X, \mu)$ the group of $\mu$-preserving invertible transformations of $(X, \mathfrak{B}, \mu)$. Throughout this paper we do not distinguish objects (measurable subsets and maps) which agree a.e. Given transformations $R_{1}, \ldots, R_{l} \in \operatorname{Aut}_{0}(X, \mu)$, we denote by $\mu_{R_{1}, \ldots, R_{l}}$ the corresponding off-diagonal measure on $X^{l}$, i.e.

$$
\mu_{R_{1}, \ldots, R_{l}}\left(B_{1} \times \cdots \times B_{l}\right):=\mu\left(R_{1} B_{1} \cap \cdots \cap R_{l} B_{l}\right) .
$$


Then $\mu_{R_{1}, \ldots, R_{l}} \in J_{l}(T)$ if and only if $R_{i} R_{j}^{-1} \in C(T)$ for all $i, j$. Recall that $C(T)$ denotes the centralizer of $T$, i.e. the group of all transformations from $\operatorname{Aut}_{0}(X, \mu)$ which commute with every $T_{g}, g \in G$. It is easy to see that the off-diagonal joinings are all ergodic.

We say that a weakly mixing $G$-action $T$ is $l$-fold pairwise independently determined ( $l$-fold PID) if any $l$-fold self-joining of $T$ which is pairwise independent (i.e. its projections on the product of any two copies of $X$ in $X^{l}$ is $\mu \times \mu$ ) must be $\underbrace{\mu \times \cdots \times \mu}_{l \text { times }}$. If $T$ is $l$-fold PID for any $l$ we say that $T$ is $P I D[\mathrm{dJR}]$. The next result follows easily from Lemma 1.1

Corollary 1.2. Let $T$ be weakly mixing. Let $H$ be a co-compact normal subgroup of $G$. Then $T$ is l-fold PID if and only if $T \uparrow H$ is l-fold PID.

We also record an important result of J. King [Ki1]: if $T$ is 4-fold PID then $T$ is PID (while stated for $\mathbb{Z}$-actions only, the result holds for arbitrary actions with the same proof).

Factors and extensions. Any $T$-invariant sub- $\sigma$-algebra $\mathfrak{F}$ of $\mathfrak{B}$ is called a factor of $T$ (or, which is the same, $T$ is an extension of $T \uparrow \mathfrak{F}$ ). The trivial sub- $\sigma$-algebra of $\mathfrak{B}$ is denoted by $\mathfrak{N}$. If $\mathfrak{B}$ and $\mathfrak{N}$ are the only factors of $T$, we call $T$ prime. Suppose that $T$ and $S$ have a common factor $\mathfrak{F}$. This means that there exists a $G$-space $(Z, \mathfrak{F}, \kappa)$ and $G$-equivariant maps $\pi_{1}: X \rightarrow Z$ and $\pi_{2}: Y \rightarrow Z$ with $\kappa=\mu \circ \pi_{1}^{-1}=\nu \circ \pi_{2}^{-1}$. Let $\mu=\int \mu_{z} d \kappa(z)$ and $\nu:=\int \nu_{z} d \kappa(z)$ denote the corresponding desintegrations. Then the measure $\mu \times_{\mathfrak{F}} \nu:=\int \mu_{z} \times \nu_{z} d \kappa(z)$ belongs to $J(T, S)$. It is called the $\mathfrak{F}$-relatively independent product of $\mu$ and $\nu$.

Let $K$ be a compact second countable group. A Borel map $\alpha: G \times X \rightarrow K$ is called a $T$-cocycle if

$$
\alpha\left(g_{2}, T_{g_{1}} x\right) \alpha\left(g_{1}, x\right)=\alpha\left(g_{2} g_{1}, x\right) \text { at a.e. } x
$$

for all $g_{1}, g_{2} \in G$. Given a closed subgroup $H \subset K$, we can define a $G$-action $T^{\alpha, H}$ on $\left(X \times K, \mu \times \lambda_{K / H}\right)$ by setting $T_{g}^{\alpha, H}(x, k H):=\left(T_{g} x, \alpha(g, x) k H\right)$, where $\lambda_{K / H}$ is Haar measure on the homogeneous space $K / H$. Then $T^{\alpha, H}$ is called an isometric extension of $T$. If $H$ is trivial, we write $T^{\alpha}$ instead of $T^{\alpha, H}$ and call it a compact group extension of $T$.

A factor $\mathfrak{F}$ of $T$ (and the extension $T \rightarrow T \uparrow \mathfrak{F}$ ) is called relatively distal if there exists a countable transfinite system $\left(\mathfrak{B}_{\alpha}\right)_{\alpha \leq \beta}$ of factors of $T$ such that $\mathfrak{B}_{0}=\mathfrak{F}, \mathfrak{B}_{\beta}=\mathfrak{B}$, the extension $T \uparrow \mathfrak{B}_{\alpha+1} \rightarrow T \uparrow \mathfrak{B}_{\alpha}$ is isometric for all $\alpha$ and $\mathfrak{B}_{\alpha}=\bigvee_{\gamma<\alpha} \mathfrak{B}_{\gamma}$ if $\alpha$ is a limit ordinal. On the other hand, a factor $\mathfrak{F}$ of $T$ (and the extension $T \rightarrow T \uparrow \mathfrak{F}$ ) is called relatively weakly mixing if the $\mathfrak{F}$-relatively independent product of $\mu$ with itself is ergodic, i.e. $\mu \times_{\mathfrak{F}} \mu \in J_{2}^{e}(T)$. Let $\mathfrak{F} \subset \mathfrak{E}$ be two factors of $T$. If the extensions $T \rightarrow T \uparrow \mathfrak{E}$ and $T \uparrow \mathfrak{E} \rightarrow T \uparrow \mathfrak{F}$ are relatively distal then the extension $T \rightarrow T \uparrow \mathfrak{F}$ is also relatively distal. If the extension $T \rightarrow T \uparrow \mathfrak{F}$ is relatively distal then so is the extension $T\lceil\mathfrak{E} \rightarrow T \uparrow \mathfrak{F}$.

Simple, quasi-simple and distally simple actions. An ergodic $G$-action $T$ is called $l$-fold simple if for each joining $\nu \in J_{l}^{e}(T)$, there exists a partition $P$ of the set $\{1, \ldots, l\}$ such that

(i) $\nu$ splits into the direct product of its marginals onto $X^{p}, p \in P$, and

(ii) the marginal of $\nu$ onto $X^{p}$ is an off-diagonal self-joining of $T$ for each $p \in P$. 
If $T$ is $l$-fold simple for any $l>1$ then we call $T$ simple. If $T$ is $l$-fold simple and $C(T) \subset\left\{T_{g} \mid g \in G\right\}$ then $T$ is said to have $l$-fold minimal self-joining property $\left(M S J_{l}\right)$. If $T$ has $\mathrm{MSJ}_{l}$ for any $l>1$ then we say that $T$ has $M S J$. As was noticed in $[\mathrm{dJR}]$, a weakly mixing 2-fold simple action is $l$-fold simple if and only if it is $l$-fold PID. Hence by [Ki1], each 4-simple action is simple. This means that we need only distinguish 3 categories of simplicity: 2-simplicity, 3-simplicity and simplicity. In general the three categories are mutually different- the corresponding examples are constructed in [Ry1] and [PRy]. However in the case of $\mathbb{Z}$-actions, two of these categories coincide: a weakly mixing 3 -fold simple transformation is simple [GlHR] (this holds also for $\mathbb{Z}^{n}$-actions). We also note that there are no examples known of transformations which are 2 -fold simple but not 3 -fold simple and this is the main open problem in this area. Moreover, if a transformation has $\mathrm{MSJ}_{2}$ but does not have $\mathrm{MSJ}_{3}$ then this transformation must be mixing [GlHR] but not 2-mixing [Ry2], answering the long standing Rokhlin's question on multiple mixing. It is interesting to note that there is not such a problem for $\mathbb{R}$-actions since each 2 -fold simple weakly mixing flow is simple [Ry2].

The following definitions introduced in [RyT] (cf. with [Ry2]) and [dJL2] for $\mathbb{Z}$ and $\mathbb{R}$-actions extend naturally to arbitrary $G$-actions as follows. An ergodic $G$ action $T$ is called 2-fold quasi-simple (2-fold $Q S$ ) if for any $\nu \in J_{2}^{e}(T) \backslash\{\mu \times \mu\}$, the two marginal extensions $(T \times T, \nu) \rightarrow(T, \mu)$ are isometric [RyT]. If, more generally, these extensions are relatively distal then $T$ is called 2-fold distally simple (2-fold $D S$ ) [dJL2]. An action is $l$-fold $Q S$ if it is 2 -fold QS and $l$-fold PID. If an action is $l$-fold QS for all $l>1$ then it is called $Q S$. In a similar way the concepts of $l$-fold DS and DS are introduced. As in the case of $l$-fold simple actions, we have only three types of quasi-simplicity: 2-fold QS, 3-fold QS and QS. The same holds for the distal simplicity. Moreover, 3-fold QS=QS for the weakly mixing $\mathbb{Z}^{n}$-actions [Ry2], [RyT] and 2-fold QS=QS for the weakly mixing flows [RyT].

We note that the class of DS-systems contains many "natural" examples. For instance, a factor of a 2 -fold simple action is 2 -fold QS. The standard $\mathrm{SL}(2, \mathbb{Z})$ action on the 2-torus $\mathbb{T}^{2}$ is 2-fold QS [Par] (but not 3-fold QS [Ry2]). A flow with the Ratner's R-property is QS [Rat], [Th1]. The flows with the R-property include the horocycle flows [Rat] and some smooth flows on 2-dimensional manifolds [FLe].

\section{2-FOLD NEAR SIMPLE ACTIONS AND VEECH THEOREM}

In this section we introduce the concept of 2-fold near simplicity (Definition 2.4) which is the main definition of this paper. Then we extend Veech theorem about the structure of factors for 2-fold simple actions to arbitrary 2-fold near simple actions (Theorem 2.6).

Let $T=\left(T_{g}\right)_{g \in G}$ be an ergodic measure preserving action of a locally compact second countable group $G$ on a standard probability space $(X, \mathfrak{B}, \mu)$. In the sequel, we will often reduce the notation $(X, \mathfrak{B}, \mu, T)$ to $X$ or $\mathfrak{B}$ or $T$.

We recall that $\operatorname{Aut}_{0}(X, \mu)$ endowed with the weak topology is a Polish group ([Ha], [Gl]). This topology is defined by

$$
R_{n} \rightarrow R \Longleftrightarrow \mu\left(R_{n} B \triangle R B\right) \rightarrow 0 \text { for each } B \in \mathfrak{B} .
$$

Moreover, $\operatorname{Aut}_{0}(X, \mu)$ is a topological 'non-commutative' $G$-module if we define the $G$-action on it via the conjugation:

$$
g \bullet S:=T_{g} S T_{g}^{-1}
$$


First of all we want to characterize the compact submodules of this module, i.e. compact $G$-invariant subgroups of $\operatorname{Aut}_{0}(X, \mu)$.

Let $K$ be a compact second countable group and let $H_{1}, H_{2}$ be two closed subgroups of $K$. We will assume that $H_{1}$ and $H_{2}$ contain no non-trivial subgroups which are normal in $K$. For every $k \in K$ and $i=1,2$, we denote by $\widetilde{k}$ the right translation of the homogeneous space $H_{i} \backslash K$ by $k$. Then the homomorphism $K \ni k \mapsto \widetilde{k^{-1}} \in \operatorname{Aut}_{0}\left(H_{i} \backslash K, \lambda_{H_{i} \backslash K}\right)$ is continuous and one-to-one. Denote by Aut $K$ the group of continuous automorphisms of $K$. It is Polish when endowed with the topology of uniform convergence.

Lemma 2.1. Let $S:\left(H_{1} \backslash K, \lambda_{H_{1} \backslash K}\right) \rightarrow\left(H_{2} \backslash K, \lambda_{H_{2} \backslash K}\right)$ be a measure space isomorphism and let $\beta \in$ Aut $K$. If $S \widetilde{k}=\widetilde{\beta(k)} S$ for all $k \in K$ then there exists $c \in K$ such that $c^{-1} H_{2} c=\beta\left(H_{1}\right)$ and $S\left(H_{1} k\right)=H_{2} c \beta(k)$ at a.e. $k \in K$. This $c$ is defined up to left multiplication with an element of $\mathrm{H}_{2}$.

Proof. Let $f: K \rightarrow K$ be a Borel map such that $f(h k)=f(k)$ for any $h \in H_{1}$ and $S\left(H_{1} k\right)=H_{2} f(k) \beta(k)$ at $\lambda_{K}$-a.e. $k \in K$. Since $S \widetilde{k}=\widetilde{\beta(k)} S$, it follows that $H_{2} f(t k)=H_{2} f(t) \beta(k)$ at a.e. $t$ for each $k \in K$. Hence there exists $c \in K$ with $H_{2} f(k)=H_{2} c \beta(k)$ at a.e. $k$. Since $f(h k)=f(k)$, we deduce that $H_{2} c \beta(k)=$ $H_{2} c \beta(h k)$. Hence $\beta\left(H_{1}\right) \subset c^{-1} H_{2} c$. Since $\beta$ preserves Haar measure $\lambda_{K}$ and $\lambda_{K}(K)=1$, we conclude that $\beta\left(H_{1}\right)=c^{-1} H_{2} c$. The final claim of the lemma is obvious.

We introduce one more kind of extensions. Denote by $\mathfrak{C}(K)$ the space of closed subgroups of $K$. In the topology introduced by Fell [Fel] this is a compact metric space with $K$ acting by conjugation. Let $\sigma: G \rightarrow$ Aut $K$ be a continuous group homomorphism and let $H: X \ni x \mapsto H_{x} \in \mathfrak{C}(K)$ and $f: G \times X \rightarrow K$ be two Borel maps such that

$$
\begin{aligned}
\sigma_{g}\left(H_{x}\right) & =f(g, x)^{-1} H_{T_{g} x} f(g, x) \text { and } \\
f\left(g_{2} g_{1}, x\right) & =f\left(g_{2}, T_{g_{1}} x\right) \sigma_{g_{2}}\left(f\left(g_{1}, x\right)\right)
\end{aligned}
$$

at a.e. $x$ for all $g, g_{1}, g_{2} \in G$. We call such $f$ a $(\sigma, H)$-twisted $T$-cocycle. Given a $(\sigma, H)$-twisted $T$-cocycle $f$, we define an action $\widetilde{T}$ of $G$ on the space $X *(H \backslash K):=$ $\bigsqcup_{x \in X}\{x\} \times H_{x} \backslash K$ with the measure $\mu * \lambda_{H \backslash K}:=\int_{X} \delta_{x} \times \lambda_{H_{x} \backslash K} d \mu(x)$ by setting

$$
\widetilde{T}_{g}\left(x, H_{x} k\right):=\left(T_{g} x, f(g, x) \sigma_{g}\left(H_{x} k\right)\right)=\left(T_{g} x, H_{T_{g} x} f(g, x) \sigma_{g}(k)\right) .
$$

We call $\widetilde{T}$ a $(\sigma, H)$-twisted isometric extension of $T$. Notice that $K$ (anti)-acts on the space $\left(X *(H \backslash K), \mu * \lambda_{H \backslash K}\right)$ by right translations along the second coordinate as follows: $k(x, H t)=(x, H t k)$. It is easy to see that $\widetilde{T}_{g} k \widetilde{T}_{g}^{-1}=\sigma_{g}(k)$ for all $k \in K$ and $g \in G$. Hence $K$ is a compact submodule of $\operatorname{Aut}_{0}\left(X *(H \backslash K), \mu * \lambda_{H \backslash K}\right)$ with respect to the $G$-action $\widetilde{T}$. Now we are going to establish a converse to that.

Given a subset $S \subset \operatorname{Aut}_{0}(X, \mu)$, we denote by $\operatorname{Fix}(S)$ the $\sigma$-algebra of subsets fixed by each transformation from $S$. Clearly, if $S$ is invariant (under the $G$ action (2-1)) then $\operatorname{Fix}(S)$ is a factor of $T$.

Proposition 2.2. Let $K$ be a compact submodule of $\operatorname{Aut}_{0}(X, \mu)$. Then $T$ is a twisted isomeric extension of its factor $\mathfrak{F}:=\operatorname{Fix}(K)$. 
Proof. It is obvious that $\mathfrak{F}$ is a factor of $T$. Considering the 'joint action' of $T$ and the 'action' of $K$ on $X$ we may assume without loss of generality that $\left(X, \mathfrak{B}_{X}, \mu\right)$ is a Borel $G \ltimes K$-space (see [Ra] for equivalence of the pointwise and Boolean approaches to locally compact dynamical systems). Since $K$ is compact, there is a Borel subset $Y \subset X$ which intersects every $K$-orbit exactly once. We consider $Y$ with the induced Borel structure as the space of $K$-orbits. Then a Borel quotient map $q: X \rightarrow Y$ sending $x \in X$ to the $K$-orbit of $x$ is well defined. This map intertwines $T$ with a Borel $G$-action on $Y$. We denote this action by $S$. Let $\nu:=\mu \circ q^{-1}$. Clearly, $S$ is $\nu$-preserving and ergodic. Since $K$ acts transitively on $q^{-1}(y)$ for each $y \in Y$, there is a Borel map $H: Y \ni y \mapsto H_{y} \in \mathfrak{C}(K)$ such that the restriction of the $K$-action to $q^{-1}(y)$ is isomorphic to the 'inverted' right $K$-action on the homogeneous space $H_{y} \backslash K$. Moreover, since a transitive action is uniquely ergodic, the conditional measure on $q^{-1}(y)$ (with respect to the projection $(X, \mu) \rightarrow(Y, \nu))$ is just the normalized Haar measure $\lambda_{H_{y} \backslash K}$. Thus there is a Borel field $\alpha: G \times Y \ni(g, y) \mapsto \alpha(g, y)$ of pointwise measure space isomorphisms

$$
\alpha(g, y):\left(H_{y} \backslash K, \lambda_{H_{y} \backslash K}\right) \rightarrow\left(H_{S_{g} y} \backslash K, \lambda_{H_{S_{g}} \backslash K}\right)
$$

such that the following are satisfied:

(i) $(X, \mu)=\left(Y *(H \backslash K), \nu * \lambda_{H \backslash K}\right)$ and $\mathfrak{F}=\left\{\bigsqcup_{y \in B}\{y\} \times H_{y} \backslash K \mid B \in \mathfrak{B}_{Y}\right\}$,

(ii) $H_{y}$ contains no non-trivial subgroups which are normal in $K$ for a.a. $y \in Y$,

(iii) $k\left(y, H_{y} t\right)=\left(y, H_{y} t k^{-1}\right)$ for all $k \in K$ and $\left(y, H_{y} t\right) \in Y *(H \backslash K)$,

(iv) $T_{g}\left(y, H_{y} t\right)=\left(S_{g} y, \alpha(g, y)\left(H_{y} t\right)\right)$ a.e. for all $g \in G$,

(v) $\alpha\left(g_{2} g_{1}, y\right)=\alpha\left(g_{2}, S_{g_{1}} y\right) \alpha\left(g_{1}, y\right)$ a.e. for all $g_{1}, g_{2} \in G$.

Since $K$ is invariant under $T$, for every $g \in G$, there exists an automorphism $\beta_{g} \in$ Aut $K$ such that $T_{g} k T_{g}^{-1}=\beta_{g}(k)$. Moreover, the map $G \ni g \mapsto \beta_{g} \in$ Aut $K$ is a continuous homomorphism. It follows from (iii) and (iv) that

$$
\alpha\left(g, S_{g}^{-1} y\right) \widetilde{k}^{-1} \alpha\left(g, S_{g}^{-1} y\right)^{-1}={\widetilde{\beta_{g}(k)}}^{-1}
$$

or, equivalently,

$$
\alpha(g, y) \widetilde{k} \alpha(g, y)^{-1}=\widetilde{\beta_{g}(k)} \text { at a.e. } y,
$$

for all $g \in G$ and $k \in K$. Hence by (ii) and Lemma 2.1, there exists a map $f: Y \times G \rightarrow K$ such that $f(g, y)^{-1} H_{S_{g} y} f(g, y)=\beta_{g}\left(H_{y}\right)$ and

$$
\alpha(g, y)\left(H_{y} t\right)=f(g, y) \beta_{g}\left(H_{y} t\right) \text { at a.e. } y,
$$

for all $g \in G$ and $k \in K$. Since $\alpha$ and $H$ are Borel and $f(g, y)$ is defined up to multiplication with an element of $H_{S_{g} y}$, we may select $f$ to be Borel. Furthermore, (v) yields that $f$ is a $(\beta, H)$-twisted $S$-cocycle.

In case $\beta=$ Id we have $K \subset C(T)$. It is easy to see that then $H$ can be chosen constant a.e., i.e. there is a subgroup $L \in \mathfrak{C}(K)$ such that $H_{y}=L$ for a.a. $y \in Y$. The corresponding $(\beta, H)$-twisted $S$-cocycle $f$ is now a usual $S$-cocycle with values in the normalizer $N_{K}(L)$ of $L$ in $K$. If $L$ is non-trivial then $N_{K}(L) \neq K$ since $L$ is not normal in $K$ by (ii). It follows that the non-constant map

$$
Y \times L \backslash K \ni(y, L k) \mapsto N_{K}(L) k \in N_{K}(L) \backslash K
$$

is invariant under $T$. This contradicts to the ergodicity of $T$. Thus if $\beta=$ Id then $H \equiv\{1\}$. However we will show in the following examples that if $\beta \neq$ Id then $H$ can be (a) constant but non-trivial or (b) essentially non-constant. 
Examples 2.3. (i) Let $K=\mathbb{T}^{2} \rtimes \mathbb{Z} / 2 \mathbb{Z}$ with the multiplication as follows

$$
(t, i)(z, j):=\left(t+(-1)^{i} z, i+j\right) .
$$

Then $H:=\{0\} \times \mathbb{Z} / 2 \mathbb{Z} \subset K$ is a closed subgroup without non-trivial normal (in $K)$ subgroups. The map $H(t, 0) \mapsto t$ identifies the quotient space $\left(H \backslash K, \lambda_{H \backslash K}\right)$ with the 2-dimensional torus $\left(\mathbb{T}^{2}, \lambda_{\mathbb{T}^{2}}\right)$. (This map is not a group homomorphism.) Then the corresponding embedding $(t, i) \mapsto \widetilde{(t, i)}$ of $K$ into $\operatorname{Aut}_{0}\left(\mathbb{T}^{2}, \lambda_{\mathbb{T}^{2}}\right)$ is given by $\widetilde{(t, i)} z:=t+(-1)^{i} z$ for all $(t, i) \in K$ and $z \in \mathbb{T}^{2}$. Let $T \in S L_{2}(\mathbb{R})$ be an ergodic automorphism of $\left(\mathbb{T}^{2}, \lambda_{\mathbb{T}^{2}}\right)$. We will consider the group $\operatorname{Aut}_{0}\left(\mathbb{T}^{2}, \lambda_{\mathbb{T}^{2}}\right)$ as a $\mathbb{Z}$-module with respect to the conjugation by $T$. We also denote by $\beta$ the following group automorphism of $K$ :

$$
\beta(t, i):=(T t, i) .
$$

It is easy to verify that $T^{n} \circ \widetilde{(t, i)} \circ T^{-n}=\widetilde{\beta(t, i)}$, i.e. $K$ considered as a transformation group on $\mathbb{T}^{2}$ is a compact submodule of $\operatorname{Aut}_{0}\left(\mathbb{T}^{2}, \lambda_{\mathbb{T}^{2}}\right)$ with $\beta \neq \mathrm{Id}$ and $H \neq\{1\}$. The latter condition just means that $K$ does not act freely. Of course, the $T$-factor $\operatorname{Fix}(K)$ is the trivial $\sigma$-algebra $\mathfrak{N}_{\mathbb{T}^{2}}$.

Notice also that $K$ has a normal subgroup $\mathbb{T}^{2}$ which embeds into $\operatorname{Aut}_{0}\left(\mathbb{T}^{2}, \lambda_{\mathbb{T}^{2}}\right)$ as a closed submodule. Moreover, $\operatorname{Fix}\left(\mathbb{T}^{2}\right)=\operatorname{Fix}(K)$. This differs from the classical situation dealing with compact subgroups in the centralizer of an ergodic dynamical system. Recall that if we have two compact subgroups $L \subsetneq K \subset C(T)$ then $\operatorname{Fix}(L) \neq \operatorname{Fix}(K)$.

(ii) (see [GS, Example 3.8]) Consider the compact Abelian group $Y:=(\mathbb{Z} / 2 \mathbb{Z})^{\mathbb{Z}}$ and let $Q$ be the Bernoulli shift of $Y$, i.e. $(Q y)_{n}=y_{n+1}$. We let $(X, \mu):=$ $\left(Y \times Y, \lambda_{Y} \times \lambda_{Y}\right)$ and $T:=Q \times Q$. Then $T$ is an ergodic transformation of $(X, \mu)$. Let $K$ denote the subgroup of $\operatorname{Aut}_{0}(X, \mu)$ generated by the transformations

$$
\theta(x, y)=(x, x+y) \text { and } \alpha(t)(x, y)=(x, y+t), t \in Y \text {. }
$$

Then $K$ is a compact submodule (with respect to $T$ ) isomorphic to $\mathbb{Z} / 2 \mathbb{Z} \times Y$. The stability group $H_{(x, y)}$ of $K$ at $(x, y) \in X$ is $\mathbb{Z} / 2 \mathbb{Z}$ generated by the transformation $\theta \cdot \alpha(x)$. Thus the map $H: X \ni(x, y) \mapsto H_{(x, y)} \in \mathfrak{C}(K)$ is not constant on any subset of positive measure in $X$.

Denote by $\Xi$ the space of probabilities on $\operatorname{Aut}_{0}(X, \nu)$. It is Polish when it is equipped with the $*$-weak topology, i.e. the smallest topology on $\Xi$ which makes the maps $\xi \mapsto \int f d \xi$ continuous for all bounded continuous functions $f$ on $\operatorname{Aut}_{0}(X, \nu)$. Let $\Xi_{T}$ stand for the subspace of $G$-invariant probabilities and let $\Xi_{T}^{e}$ denote the subspace of ergodic $G$-invariant probabilities on $\operatorname{Aut}_{0}(X, \nu)$. Then $\Xi_{T}$ is a closed convex subset of $\Xi$ and $\Xi_{T}^{e}$ is the set of extremal points of $\Xi_{T}$. By [Va], $\Xi_{T}^{e}$ is a Borel subset of $\Xi_{T}$.

The centralizer $C(T)=\{R \mid g \bullet R=R\}$ of $T$ embeds into $\Xi_{T}^{e}$ as the closed subset of $G$-invariant Dirac $\delta$-measures. More generally, if $\xi \in \Xi_{T}^{e}$ and $\operatorname{Supp} \xi$ is finite, say $\operatorname{Supp} \xi=\left\{S_{1}, \ldots, S_{n}\right\}$, then $T_{g} S_{i} T_{g}^{-1}=S_{\sigma_{g}(i)}$, where $(g, i) \mapsto \sigma_{g}(i)$ is a transitive action of $G$ on the finite set $\{1, \ldots, n\}$.

Notice that there are two natural continuous operations on $\Xi_{T}$ : the convolution $\star: \Xi_{T} \times \Xi_{T} \rightarrow \Xi_{T}$ and an involution $*: \Xi_{T} \rightarrow \Xi_{T}$ given by $\xi^{*}(A):=\xi\left(A^{-1}\right)$. Clearly, $\xi^{*} \in \Xi_{T}^{e}$ whenever $\xi \in \Xi_{T}^{e}$. Let $\mathcal{P}\left(\Xi_{T}^{e}\right)$ denote the space of all probabilities 
on $\Xi_{T}^{e}$. It follows from [Va] that there is a Borel isomorphism (ergodic decomposition)

$$
\pi: \Xi_{T} \ni \xi \mapsto \pi(\xi) \in \mathcal{P}\left(\Xi_{T}^{e}\right)
$$

such that $\xi=\int \eta d(\pi(\xi))(\eta)$ and $\pi(\xi)=\delta_{\xi}$ if $\xi \in \Xi_{T}^{e}$. Given $\xi, \nu \in \Xi_{T}^{e}$, the convolution $\xi \star \nu$ does not necessarily belong to $\Xi_{T}^{e}$. We will denote the measure $\pi(\xi \star \nu)$ by $\lambda_{\xi, \eta}$.

Given $\xi \in \Xi_{T}$, we let

$$
\operatorname{Fix}(\xi):=\left\{A \in \mathfrak{B} \mid \mu(A \triangle S A)=0 \text { for } \xi \text {-a.a. } S \in \operatorname{Aut}_{0}(X, \mu)\right\} .
$$

It is easy to verify that $\operatorname{Fix}(\xi)$ is a factor of $T$. Notice that $\operatorname{Fix}(\xi)$ depends only on the support of $\xi$. Denote by $H$ the closure of the subgroup generated by Supp $\xi$. Notice that $H$ is $G$-invariant and $\operatorname{Fix}(\xi)=\operatorname{Fix}(H)$. Given a subset $\Delta \subset \Xi_{T}$, we also let $\operatorname{Fix}(\Delta):=\bigcap_{\xi \in \Delta} \operatorname{Fix}(\xi)$.

Now for any $\xi \in \Xi_{T}$, we define a measure on $(X \times X, \mathfrak{B} \times \mathfrak{B})$ by setting

$$
\mu_{\xi}(A \times B):=\int_{\operatorname{Aut}_{0}(X, \mu)} \mu(A \cap S B) d \xi(S) .
$$

Then $\mu_{\xi}$ is a self-joining of $T$. The 'map' $\Lambda:(x, \theta) \mapsto(x, \theta x)$ conjugates the diagonal $G$-action on $\left(X \times \operatorname{Aut}_{0}(X, \mu), \mu \times \xi\right)$ with $\left(T \times T, \mu_{\xi}\right)$. We encounter here with a problem that the map under question is not well defined formally-notice that $\theta x$ has no sense. However $\Lambda$ is well defined on the underlying $\sigma$-Boolean algebras and hence admits a pointwise realization.

Notice that $\mu_{\xi}$ is ergodic whenever $T$ is weakly mixing and $\xi \in \Xi_{T}^{e}$.

\section{Definition 2.4.}

(i) We call $T$ 2-fold near simple if $J_{2}^{e}(T)=\left\{\mu_{\xi} \mid \xi \in \Xi_{T}^{e}\right\} \cup\{\mu \times \mu\}$,

(ii) We say that $T$ has 2-fold near minimal self-joinings (near $M S J_{2}$ ) if $T$ is 2-fold near simple and $\operatorname{Supp} \xi \subset\left\{T_{g} \mid g \in G\right\}$ for any $\xi \in \Xi_{T}^{e}$.

Notice that according to this definition each 2-fold near simple action is automatically weakly mixing (see also Remark 2.8 at the end of this section). We also note that every weakly mixing 2 -fold simple action is 2 -fold near simple.

Remark 2.5. In case $G$ is countable and discrete, we denote by $\mathrm{FC}(G)$ the subset of elements $g$ whose conjugacy class $g^{*} \subset G$ is finite. It is clear that $\operatorname{FC}(G)$ is a normal subgroup of $G$. Then $T$ has near $\mathrm{MSJ}_{2}$ if and only if

$$
J_{2}^{e}(T)=\left\{\frac{1}{\# g^{*}} \sum_{h \in g^{*}} \mu_{T_{h}} \mid g \in \mathrm{FC}(G)\right\} \cup\{\mu \times \mu\},
$$

where $\mu_{T_{h}}(A \times B):=\mu\left(A \cap T_{h} B\right), A, B \in \mathfrak{B}$.

Denote by $I(T)$ the smallest closed subgroup of $\operatorname{Aut}_{0}(X, \mu)$ which contains the supports of all $\xi \in \Xi_{T}^{e}$. Clearly, $I(T)$ is $G$-invariant (with respect to (2-1)), i.e. $I(T)$ is a closed submodule of $\operatorname{Aut}_{0}(X, \mu)$. Moreover, $I(T) \supset C(T)$. Let $T$ be 2 -fold near simple. Then $T$ is 2 -fold simple if and only if $I(T)=C(T)$.

Let $\mathfrak{F}$ be a factor of $T$. We set

$$
\Xi_{T}^{e}(\mathfrak{F}):=\left\{\xi \in \Xi_{T}^{e} \mid \operatorname{Fix}(\xi) \supset \mathfrak{F}\right\}
$$

Clearly, $\Xi_{T}^{e}(\mathfrak{F})$ is a closed subset of $\Xi_{T}^{e}$. Notice also that $\Xi_{T}^{e}(\mathfrak{B})=\left\{\delta_{\mathrm{Id}}\right\}$. We also denote by $I(T, \mathfrak{F})$ the smallest subgroup of $\operatorname{Aut}_{0}(X, \mu)$ which contains the supports of all $\xi \in \Xi_{T}^{e}(\mathfrak{F})$. Then $\operatorname{Fix}\left(\Xi_{T}^{e}(\mathfrak{F})\right)=\operatorname{Fix}(I(T, \mathfrak{F}))$.

The following theorem is an analogue of Veech theorem on factors of 2-fold simple actions (see [Ve, Theorem 1.2] or [dJR, Theorem 3.1]). 
Theorem 2.6. Let $T$ be 2-fold near simple and let $\mathfrak{F}$ be a non-trivial factor of $T$. Then $I(T, \mathfrak{F})$ is a compact submodule of $I(T)$ and $\mathfrak{F}=\operatorname{Fix}(I(T, \mathfrak{F}))$.

Proof. It is easy to see that $I(T, \mathfrak{F})$ is a submodule of $I(T)$. Consider the ergodic decomposition of the $\mathfrak{F}$-relatively independent product $\mu \times_{\mathfrak{F}} \mu$ of $\mu$ with itself:

$$
\mu \times_{\mathfrak{F}} \mu=\int_{\Xi_{T}^{e}} \mu_{\xi} d \kappa(\xi)
$$

We used the fact that $T$ is 2-fold near simple and that $\mu \times_{\mathfrak{F}} \mu \nsucc \mu \times \mu$ since $\mathfrak{F}$ is nontrivial. Notice that a subset $B \in \mathfrak{B}$ belongs to $\mathfrak{F}$ if and only if $\mu \times_{\mathfrak{F}} \mu\left(B \times B^{c}\right)=0$. The latter is equivalent to $\mu_{\xi}\left(B \times B^{c}\right)=0$ for $\kappa$-a.a. $\xi \in \Xi_{T}^{e}$. However $\mu_{\xi}\left(B \times B^{c}\right)=$ 0 if and only if $B \in \operatorname{Fix}(\xi)$. Hence choosing a countable dense in $\mathfrak{F}$ family $B_{i}$, we obtain that $\kappa\left(\Xi_{T}^{e}(\mathfrak{F})\right)=1$ and hence

$$
\mu \times_{\mathfrak{F}} \mu=\int_{\Xi_{T}^{e}(\mathfrak{F})} \mu_{\xi} d \kappa(\xi)
$$

Clearly, $\mathfrak{F} \subset \operatorname{Fix}\left(\Xi_{T}^{e}(\mathfrak{F})\right)$. The converse inclusion follows from $(2-2)$. Hence $\mathfrak{F}=$ $\operatorname{Fix}\left(\Xi_{T}^{e}(\mathfrak{F})\right)$. Now we let $\widetilde{\kappa}:=\int_{\Xi_{T}^{e}(\mathfrak{F})} \xi d \kappa(\xi)$. Of course, $\widetilde{\kappa} \in \Xi_{T}$ and $\operatorname{Supp} \widetilde{\kappa} \subset$ $I(T, \mathfrak{F})$.

It is well known and easy to check that if $S A=A$ for all $A \in \mathfrak{F}$ then

$$
\left(\mu \times_{\mathfrak{F}} \mu\right) \circ(\operatorname{Id} \times S)=\mu \times_{\mathfrak{F}} \mu .
$$

Hence for any $\nu \in \Xi_{T}^{e}(\mathfrak{F})$,

$$
\int_{\operatorname{Aut}_{0}(X, \mu)}\left(\mu \times_{\mathfrak{F}} \mu\right) \circ(\operatorname{Id} \times S) d \nu(S)=\mu \times_{\mathfrak{F}} \mu .
$$

On the other hand, it is easy to verify that

$$
\int_{\operatorname{Aut}_{0}(X, \mu)} \mu_{\xi} \circ(\operatorname{Id} \times S) d \nu(S)=\mu_{\xi \star \nu}
$$

Hence substituting (2-2) into (2-3) we derive that

$$
\mu \times_{\mathfrak{F}} \mu=\int_{\Xi_{T}^{e}(\mathfrak{F})} \mu_{\xi \star \nu} d \kappa(\xi)
$$

for each $\nu \in \Xi_{T}^{e}(\mathfrak{F})$. It is easy to see that $\xi \star \nu \in \Xi_{T}(\mathfrak{F})$ whenever $\xi, \nu \in \Xi_{T}^{e}(\mathfrak{F})$. Hence in the ergodic decomposition $\xi \star \nu=\int \eta d \lambda_{\xi, \nu}(\eta)$, we have $\lambda_{\xi, \nu}\left(\Xi_{T}^{e}(\mathfrak{F})\right)=1$. Therefore (2-4) implies

$$
\mu \times_{\mathfrak{F}} \mu=\int_{\Xi_{T}^{e}(\mathfrak{F})} \int_{\Xi_{T}^{e}(\mathfrak{F})} \mu_{\eta} d \lambda_{\xi, \nu}(\eta) d \kappa(\xi)=\int_{\Xi_{T}^{e}(\mathfrak{F})} \mu_{\eta} d\left(\int_{\Xi_{T}^{e}(\mathfrak{F})} \lambda_{\xi, \nu} d \kappa(\xi)\right)(\eta) .
$$

By the uniqueness of the ergodic decomposition, we obtain 


$$
\kappa=\int_{\Xi_{T}^{e}(\mathfrak{F})} \lambda_{\xi, \nu} d \kappa(\xi)
$$

for each $\nu \in \Xi_{T}^{e}(\mathfrak{F})$. This yields

$$
\begin{aligned}
\widetilde{\kappa} \star \nu & =\int \xi \star \nu d \kappa(\xi) \\
& =\iint \eta d \lambda_{\xi, \nu}(\eta) d \kappa(\xi) \\
& =\int \eta d\left(\lambda_{\xi, \nu} d \kappa(\xi)\right)(\eta) \\
& =\int \eta d \kappa(\eta) .
\end{aligned}
$$

Thus $\widetilde{\kappa} \star \nu=\widetilde{\kappa}$ for each $\nu \in \Xi_{T}^{e}(\mathfrak{F})$. This implies in turn that $\widetilde{\kappa} \star \widetilde{\kappa}=\widetilde{\kappa}$. Then by $[\mathrm{Pa}]$, Supp $\widetilde{\kappa}$ is a compact group and $\widetilde{\kappa}$ is normalized Haar measure on it. Now the fact $\widetilde{\kappa} \star \nu=\widetilde{\kappa}$ implies that $\operatorname{Supp} \widetilde{\kappa}$ contains $\operatorname{Supp} \nu$ for each $\nu \in \Xi_{T}^{e}(\mathfrak{F})$. Hence $\operatorname{Supp} \widetilde{\kappa}=I(T, \mathfrak{F})$. It remains to use the fact that $\operatorname{Fix}(I(T, \mathfrak{F}))=\operatorname{Fix}\left(\Xi_{T}^{e}(\mathfrak{F})\right)=$ $\mathfrak{F}$.

Corollary 2.7. Let $T$ be free and have near $M S J_{2}$. Then the map $K \mapsto \operatorname{Fix}(K)$ is a one-to-one correspondence between the compact normal subgroups of $G$ and the non-trivial factors of $T$.

Proof. In view of Theorem 2.6, we only need to show that the map under question is one-to-one. This follows from the fact that $K$ acts freely (since $T$ is free).

In [Da3], for any infinite countable sum $G$ of finite groups, we constructed an uncountable family of mixing rank-one free $G$-actions which have near $\mathrm{MSJ}_{2}$ (in fact, near MSJ of any order). Thus Corollary 2.7 provides a description of the factors of such actions. Notice that the classical Veech theorem is not applicable in this situation if $G$ is non-commutative since no one free $G$-action is 2-fold simple.

Remark 2.8. It seems natural to to extend Definition 2.4 to non-weakly mixing actions $T$ as follows: $T$ is 2-fold near simple if $J_{2}^{e}(T) \subset\left\{\mu_{\xi} \mid \xi \in \Xi_{T}^{e}\right\}$. Then taking $\mathfrak{F}:=\mathfrak{N}$ and repeating the proof of Theorem 2.6 almost verbally we obtain that $I(T, \mathfrak{N})=I(T)=: K$ is compact and $\operatorname{Fix}(K)=\mathfrak{N}$. If $G$ is Abelian then we obtain that the closure of $\left\{T_{g} \mid g \in G\right\}$ in $\operatorname{Aut}_{0}(X, \mu)$ is a compact Abelian group, say $H$. Hence without loss of generality we may assume that the following holds: $(X, \mu)=\left(H, \lambda_{H}\right)$, there exists a continuous homomorphism $\phi: G \rightarrow H$ whose range is dense in $H$ and $T_{g} h=\phi(g) h$ for all $g \in G$ and $h \in H$. It follows that $T$ is 2-fold simple and hence $H=K$. However, if $G$ is not Abelian then the situation is not so clear for us. By Proposition 2.2, $T$ is a twisted isometric extension of the trivial one-point $G$-action. This means that $(X, \mu)=\left(H \backslash K, \lambda_{H \backslash K}\right)$ and $T_{g}(H k)=H f(g) \sigma_{g}(k)$, where

- $H \subset K$ is a closed subgroup without nontrivial $G$-normal subgroups,

- $G \ni g \mapsto \sigma_{g} \in \operatorname{Aut}_{H}(K)$ is a continuous homomorphism and

- $f: G \rightarrow K$ is a Borel (and hence continuous) skew homomorphism, i.e. $f\left(g_{2} g_{1}\right)=f\left(g_{2}\right) \sigma_{g_{2}}\left(f\left(g_{1}\right)\right)$ for all $g_{1}, g_{2} \in G$. 
In other words, $T$ is an algebraic (homogeneous) factor of the affine $G$-action $\widetilde{T}$ on $\left(K, \lambda_{K}\right)$ given by $\widetilde{T}_{g} k:=f(g) \sigma_{g}(k)$. However, not every such action is 2 -fold near simple. (They can be Bernoullian.) We do not know a 'good' criterium of simplicity for algebraic factors of affine actions of non-Abelain locally compact groups. That is why we included the assumption of weak mixing into Definition 2.4.

\section{Higher ORDER NEAR SIMPLICITY AND NEAR MSJ}

In this section we define the properties of near simplicity and near MSJ of higher orders (Definition 3.1). They extend the corresponding properties of simplicity and MSJ of higher order from [dJR]. As in [dJR], we investigate whether these properties are inherited by co-compact subactions (Proposition 3.2), factors (Propositions 3.4, 3.6) and compact group extensions (Proposition 3.2).

As above, $T$ is an ergodic $G$-action on $(X, \mathfrak{B}, \mu)$. For $l>0$, we denote by $\mathcal{A}^{l}$ the $l$-th Cartesian power of the $G$-module $\operatorname{Aut}_{0}(X, \mu)$. Let $\Xi_{T, l}$ and $\Xi_{T, l}^{e}$ stand for the space of $G$-invariant and ergodic $G$-invariant (respectively) probabilities on $\mathcal{A}^{l}$. Given a measure $\xi \in \Xi_{T, l}$ and a partition $P$ of $\{1, \ldots, l\}$, we define a measure $\mu_{\xi, P}$ on $\left(X^{l}, \mathfrak{B}^{\otimes l}\right)$ by setting

$$
\mu_{\xi, P}\left(A_{1} \times \cdots \times A_{l}\right)=\int_{\mathcal{A}_{l}} \prod_{p \in P} \mu\left(\bigcap_{i \in p} S_{i} A_{i}\right) d \xi\left(S_{1}, \ldots, S_{l}\right) .
$$

It is easy to see that $\mu_{\xi, P} \in J_{l}(T)$. Let $P_{0}$ denotes the trivial partition. Then every 'marginal' extension $\left(X^{l}, \mu_{\xi, P_{0}}, T^{(l)}\right) \rightarrow(X, \mu, T)$ splits into the direct product $(X, \mu, T) \times\left(\mathcal{A}^{l}, \xi, \bullet\right) \rightarrow(X, \mu, T)$, where $T^{(l)}$ denotes the direct product of $l$ copies of $T$ and $\bullet$ denotes the $G$-action determined by the module structure on $\mathcal{A}^{l}$. The converse to the splitting isomorphism under question is given by

$$
X \times \mathcal{A}^{l} \ni\left(x, S_{1}, \ldots, S_{l}\right) \mapsto\left(S_{1} x, \ldots, S_{l} x\right) \in X^{l} .
$$

If $P$ is not trivial, we select a subset $Q \subset\{1, \ldots, l\}$ which meets every atom of $P$ exactly once. Then the 'marginal' extension $\left(X^{l}, \mu_{\xi, P}, T^{(l)}\right) \rightarrow(X, \mu, T)^{Q}$ splits into the direct product $(X, \mu, T)^{Q} \times\left(\mathcal{A}^{l}, \xi, \bullet\right) \rightarrow(X, \mu, T)^{Q}$. We leave the exact formula for the splitting isomorphism to the reader.

\section{Definition 3.1.}

(i) We say that $T$ is l-fold near simple if

$$
J_{l}^{e}(T)=\left\{\mu_{\xi, P} \mid \xi \in \Xi_{T, l}^{e}, P \text { is a partition of }\{1, \ldots, l\}\right\} .
$$

(ii) We say that $T$ is near simple if $T$ is $l$-fold near simple for any $l>1$.

(iii) If $T$ is $l$-fold near simple (near simple) and $I(T) \subset\left\{T_{g} \mid g \in G\right\}$ then we say that $T$ has near $M S J_{l}$ (near $M S J$ ).

Notice that in case $l=2$ this definition of 2 -fold near simplicity looks a bit different-more symmetric - from what we defined in Section 2. However the two definitions are equivalent since the following equivariant map

$$
\pi: \mathcal{A}^{2} \ni\left(S_{1}, S_{2}\right) \mapsto S_{1}^{-1} S_{2} \in \mathcal{A}
$$

takes $\Xi_{T, 2}^{e}$ onto $\Xi_{T}^{e}$ and $\mu_{\xi, P_{0}}=\mu_{\xi \circ \pi^{-1}}$ for each $\xi \in \Xi_{T, 2}^{e}$. Notice also that if $T$ is $l$-fold near simple then it is $l$-fold simple if and only if $I(T)=C(T)$.

Let $H$ be a co-compact normal subgroup in $G$. Denote by $s: H \backslash G \rightarrow G$ a Borel cross-section of the natural projection $G \rightarrow H \backslash G$. 
Proposition 3.2. Let $T$ be weakly mixing. Then $T$ is l-fold near simple if and only if the $H$-subaction $T \uparrow H:=\left(T_{g}\right)_{g \in H}$ is l-fold near simple. Moreover,

$$
\Xi_{T, l}^{e}=\left\{\int_{H \backslash G} \eta \circ s(t) d \lambda_{H \backslash G}(t) \mid \eta \in \Xi_{T\lceil H, l}^{e}\right\}
$$

and hence $I(T)=I(T \uparrow H)$.

Proof. Suppose first that $T$ is $l$-fold near simple. Let $\nu \in J_{l}^{e}(T \uparrow H)$. Then the measure $\widehat{\nu}:=\int_{H \backslash G} \nu \circ T_{s(t)}^{(l)} d \lambda_{H \backslash G}(t)$ is $T^{(l)}$-invariant and ergodic by Lemma 1.1. Since the marginals of $\nu \circ T_{s(t)}^{(l)}$ are all equal to $\mu$ for any $t \in H \backslash G$, it follows that $\widehat{\nu} \in J_{l}^{e}(T)$. Since $T$ is $l$-fold near simple, there exist a measure $\xi \in \Xi_{T, l}^{e}$ and a partition $P$ of $\{1, \ldots, l\}$ with $\widehat{\nu}=\mu_{\xi, P}$. By Lemma 1.1, there exists a measure $\eta \in \Xi_{T\lceil H, l}^{e}$ such that $\xi=\int_{H \backslash G} \eta \circ s(t) d \lambda_{H \backslash G}(t)$. Hence

$$
\mu_{\xi, P}=\int_{H \backslash G} \mu_{\eta \circ s(t), P} d \lambda_{H \backslash G}(t)=\int_{H \backslash G} \mu_{\eta, P} \circ T_{s(t)}^{(l)} d \lambda_{H \backslash G}(t) .
$$

Since $H$ is normal, it follows from Lemma 1.1 and the uniqueness of the ergodic decomposition that $\nu=\mu_{\eta \circ g, P}$ for some $g \in G$. Hence $T \uparrow H$ is $l$-fold near simple.

Conversely, if $T \uparrow H$ is $l$-fold near simple then by Lemma 1.1, any measure $\lambda \in J_{l}^{e}(T)$ can be written as $\lambda=\widehat{\mu_{\eta, P}}$ for some $\eta \in \Xi_{T \uparrow H, l}^{e}$ and a partition $P$ of $\{1, \ldots, l\}$. It remains to notice that $\widehat{\mu_{\eta, P}}=\mu_{\widehat{\eta}, P}$.

We apply these results to simple actions. Notice that the "only if" part of the following corollary was established, in fact, in [dJR, Theorem 6.1].

Corollary 3.3. Let $T$ be weakly mixing. Then $T$ is l-fold simple if and only if $T \uparrow H$ is l-fold simple plus $C(T)=C(T \uparrow H)$.

Proof. We first notice that

$$
C(T) \subset C(T \uparrow H) \subset I(T \uparrow H)=I(T) \supset C(T) .
$$

Now, if $T$ is $l$-fold simple then $I(T)=C(T)$ and hence $C(T)=C(T \uparrow H)=I(T \uparrow$ $H)$. Hence $T \nmid H$ is $l$-fold simple. The converse is established in a similar way.

Let $\mathfrak{F}$ be a factor of $T$. We let $\operatorname{Aut}_{0}(\mathfrak{F}):=\left\{S \in \operatorname{Aut}_{0}(X, \mu) \mid S \mathfrak{F}=\mathfrak{F}\right\}$. Clearly, $\operatorname{Aut}_{0}(\mathfrak{F})$ is a closed subgroup in $\operatorname{Aut}_{0}(X, \mu)$. Moreover, let $\operatorname{Aut}_{0}(\mathfrak{F}, \mu\lceil\mathfrak{F})$ denote the automorphism group of the quotient space $(X, \mathfrak{F}, \mu \uparrow \mathfrak{F})$. The restriction map

$$
\operatorname{Aut}_{0}(\mathfrak{F}) \ni S \mapsto S \uparrow \mathfrak{F} \in \operatorname{Aut}_{0}(\mathfrak{F}, \mu\lceil\mathfrak{F})
$$

is a $G$-equivariant homomorphism. Given a measure $\eta$ on $\operatorname{Aut}_{0}(\mathfrak{F})$, we denote by $\eta\lceil\mathfrak{F}$ the image of $\eta$ under this homomorphism.

Proposition 3.4. Let $T$ be l-fold near simple and let $\mathfrak{F}=\operatorname{Fix}(K)$ for a compact submodule $K \subset \operatorname{Aut}_{0}(X, \mu)$. If $K$ is normal in $I(T)$ then $\mathfrak{F}$ is l-fold near simple and $\Xi_{\mathfrak{F}}^{e}=\left\{\xi\left\lceil\mathfrak{F} \mid\right.\right.$ for all $\left.\xi \in \Xi_{T}^{e}\right\}$. Hence $I(\mathfrak{F})=I(T)\lceil\mathfrak{F}$.

Proof. We first consider the case $l=2$. Given any $\xi \in \Xi_{T}^{e}$, we have $S K S^{-1}=K$ for each $S \in \operatorname{Supp} \xi \subset I(T)$ by the assumption on $K$. Hence $\operatorname{Supp} \xi \subset \operatorname{Aut}_{0}(\mathfrak{F})$ and the 
restriction $\xi\left\lceil\mathfrak{F}\right.$ is well defined. Moreover, $\left(\mu\lceil\mathfrak{F})_{\xi \mid \mathfrak{F}}=\mu_{\xi} \uparrow(\mathfrak{F} \otimes \mathfrak{F}) \in J_{2}^{e}(\mathfrak{F})\right.$. On the other hand, take any $\nu \in J_{2}^{e}(\mathfrak{F}), \nu \neq \mu \uparrow \mathfrak{F} \times \mu \uparrow \mathfrak{F}$. It is a standard fact of the joining theory that there exists $\rho \in J_{l}^{e}(T)$ with $\nu=\rho \uparrow(\mathfrak{F} \otimes \mathfrak{F})$ ( $\rho$ is a component in the ergodic decomposition of the relatively independent extension of $\nu$ ). Since $T$ is 2-fold near simple and $\rho \neq \mu \times \mu$, we obtain that $\rho=\mu_{\xi}$ for some $\xi \in \Xi_{T}^{e}$. Hence

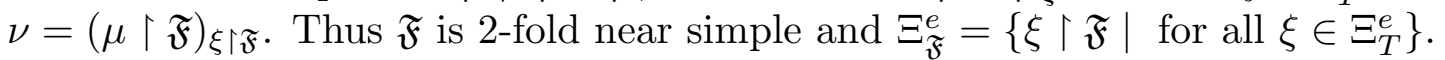

Now consider the case $l>2$. Then for any $\nu \in J_{l}^{e}(\mathfrak{F})$, one can find a measure $\rho \in J_{l}^{e}(T)$ such that $\rho \nmid \mathfrak{F}^{\otimes l}=\nu$. Hence $\rho=\mu_{\xi, P}$ for some $\xi \in \Xi_{T, l}^{e}$ and a partition $P$ of $\{1, \ldots, l\}$. We note that such $\xi$ is not unique. For each $p \in P$, we put $j_{p}:=\min _{i \in p} i$. Since $\mu\left(\bigcap_{i \in p} S_{i} A_{i}\right)=\mu\left(\bigcap_{i \in p} S_{j_{p}}^{-1} S_{i} A_{i}\right)$, there exists $\bar{\xi} \in \Xi_{T, l}^{e}$ such that $\mu_{\bar{\xi}, P}=\mu_{\xi, P}$ and the marginals of $\bar{\xi}$ onto the $j_{p}$-coordinate is $\delta_{\text {Id }}$ for each $p \in P$. Now it is easy to see that the marginals of $\bar{\xi}$ onto any other coordinate belong to $\Xi_{T}^{e}$. Hence we deduce from the first part of the proof that $\operatorname{Supp} \bar{\xi} \subset \operatorname{Aut}_{0}(\mathfrak{F})^{l}$. Hence $\nu=\mu_{\bar{\xi}, P}\left\lceil\mathfrak{F}^{\otimes l}=\left(\mu\lceil\mathfrak{F})_{\bar{\xi} \mid \mathfrak{F} \otimes l, P}\right.\right.$. This implies that $\mathfrak{F}$ is $l$-fold near simple.

The above result yields the 'if' part of [dJR, Corollary 3.6]:

Corollary 3.5. If $T$ is l-fold simple and $K$ is a compact normal subgroup in $C(T)$ then the factor $\mathfrak{F}:=\operatorname{Fix}(K)$ of $T$ is also l-fold simple. Moreover, $C(T) \subset \operatorname{Aut}_{0}(\mathfrak{F})$ and $C(T \uparrow \mathfrak{F})=C(T) \uparrow \mathfrak{F}$.

It was also shown in [dJR, Corollary 3.6] that if $T$ is simple and $K$ a non-normal compact subgroup of $C(T)$ then the factor $\operatorname{Fix}(K)$ of $T$ is not simple. We will show that this factor is not even near simple.

Proposition 3.6. Let $T$ be weakly mixing and 2-fold simple. Let $K$ be a nonnormal compact subgroup of $C(T)$. Then the factor $\mathfrak{F}:=\operatorname{Fix}(K)$ is not 2-fold near simple.

Proof. We will prove this by contradiction. Suppose that $\mathfrak{F}$ is 2 -fold near simple. Take any transformation $R \in C(T)$ such that $R K R^{-1} \neq K$. Then either

(i) $\mu_{R} \uparrow(\mathfrak{F} \otimes \mathfrak{F})=(\mu\lceil\mathfrak{F}) \times(\mu\lceil\mathfrak{F})$ or

(ii) there exists a measure $\xi \in \Xi_{\mathfrak{F}}^{e}$ such that $\mu_{R} \uparrow(\mathfrak{F} \otimes \mathfrak{F})=(\mu \uparrow \mathfrak{F})_{\xi}$.

We first show that (i) is impossible. Indeed, the diagram

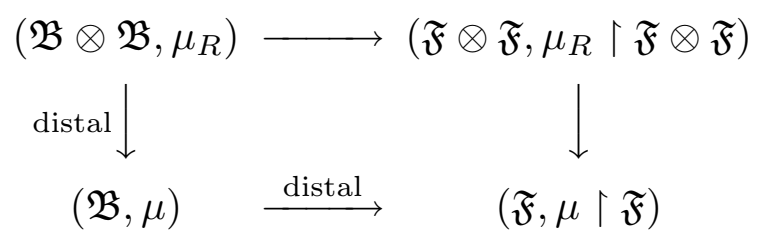

commutes. Hence the extension $\left(\mathfrak{B} \otimes \mathfrak{B}, \mu_{R}\right) \rightarrow(\mathfrak{F}, \mu \uparrow \mathfrak{F})$ is distal as a composition of two distal extensions. It follows that its 'subextension' $\left(\mathfrak{F} \otimes \mathfrak{F}, \mu_{R}\lceil\mathfrak{F} \otimes \mathfrak{F}) \rightarrow\right.$ $(\mathfrak{F}, \mu\lceil\mathfrak{F})$ is also distal. Hence if (i) holds then we deduce that the factor $\mathfrak{F}$ is distal. Since $T$ is a compact group extension of $\mathfrak{F}$, it follows that $T$ is distal, a contradiction. Thus (ii) holds and therefore

$$
\mu(A \cap R B)=\int_{\mathrm{Aut}_{0}(\mathfrak{F}, \mu \mid \mathfrak{F})} \mu(A \cap S B) d \xi(S)
$$

for all $A, B \in \mathfrak{F}$. It follows immediately that $\xi$ is a Dirac $\delta$-measure supported at a transformation $\widetilde{R} \in \operatorname{Aut}_{0}(\mathfrak{F}, \mu \uparrow \mathfrak{F})$. Hence $\widetilde{R} \in C(T \uparrow \mathfrak{F})$ and $\widetilde{R}=R \uparrow \mathfrak{F}$. Therefore $R \in \operatorname{Aut}_{0}(\mathfrak{F})$ and we have $R K R^{-1}=K$ by [dJR, Corollary 3.3], a contradiction. 
Our next aim is to investigate near simplicity of weakly mixing compact group extensions of actions with near MSJ. We first state an auxiliary lemma. Let $K$ be a compact second countable group and $\alpha: G \times X \rightarrow K$ a Borel cocycle of $T$. Denote by $T^{\alpha}$ the corresponding group extension of $T$. For $k \in K$, let $R_{k}: X \times K \rightarrow X \times K$ denote the right translation by $k$ along $K$.

Lemma 3.7. Let $\lambda$ and $\rho$ be two ergodic $T^{\alpha}$-invariant measures on $X \times K$. If the projection of $\lambda$ and $\rho$ onto $X$ are both equal to $\mu$ then there is $k \in K$ such that $\lambda=\rho \circ R_{k}$.

Proof. By [Zi, Corollary 3.8], there exists a closed subgroup $H \subset K$, a Borel map $a: X \rightarrow K$ and a Borel cocycle $\beta: G \times X \rightarrow H$ such that

$$
a\left(T_{g} x\right) \alpha(g, x) a(x)^{-1}=\beta(g, x) \text { at } \mu \text {-a.e. } x \text { for every } g \in G
$$

and the $G$-action $T^{\beta}$ on the space $\left(X \times H, \mu \times \lambda_{H}\right)$ is ergodic. We define a map $\Lambda: X \times K \rightarrow X \times K$ by setting $\Lambda(x, k):=(x, a(x) k)$. Then $\Lambda T_{g}^{\alpha} \Lambda^{-1}=T_{g}^{\beta}$ for all $g \in G$. The measure $\lambda^{*}:=\lambda \circ \Lambda^{-1}$ is $T^{\beta}$-invariant and ergodic. Since the map $X \times K \ni(x, k) \mapsto H k \in H \backslash G$ is $\lambda^{*}$-a.e. invariant under $T^{\beta}$, there exists $k \in K$ with $\lambda^{*}\left(X \times H k^{-1}\right)=1$. Hence $\lambda^{*} \circ R_{k}(X \times H)=1$. Now it follows from a version of Furstenberg's lemma on the relatively unique ergodicity of compact group extensions (see [dJR, Lemma 5.1]) that $\lambda^{*} \circ R_{k}=\mu \times \lambda_{H}$. In a similar way, $\rho^{*} \circ R_{k^{\prime}}=\mu \times \lambda_{H}$ for some $k^{\prime} \in K$. It remains to notice that $\Lambda$ commutes with $R_{t}$ for any $t \in K$.

Suppose now that $T$ is effective, i.e. the homomorphism $G \ni g \mapsto T_{g} \in \mathcal{A}$ is one-to-one. It was shown in the proof of Proposition 3.4 that for each $l$-fold near simple $G$-action $T$,

$$
J_{l}^{e}(T)=\left\{\mu_{\xi, P} \mid \xi \in \Xi_{T, l}^{e} \text { with } \operatorname{Supp} \xi \subset I(T)^{l}, P \text { is a partition of }\{1, \ldots, l\}\right\} .
$$

Hence if $T$ has near $\mathrm{MSJ}_{l}$ and $\nu=\mu_{\xi, P}$, we may assume without loss of generality that $\xi$ is supported on the sub- $G$-module $\left\{T_{g_{1}} \times \cdots \times T_{g_{l}} \mid g_{1}, \ldots, g_{l} \in G\right\} \subset \mathcal{A}^{l}$. Denote by $\xi^{\alpha}$ the image of $\xi$ under the map adjoint to the following $G$-module homomorphism

$$
T_{g_{1}} \times \cdots \times T_{g_{l}} \mapsto T_{g_{1}}^{\alpha} \times \cdots \times T_{g_{l}}^{\alpha} \in \operatorname{Aut}_{0}\left(X \times K, \mu \times \lambda_{K}\right)^{l} .
$$

This homomorphism is well defined since $T$ is effective. Since $\xi$ is ergodic, it follows that $\xi^{\alpha} \in \Xi_{T^{\alpha}, l}^{e}$. We also observe that $\Xi_{T^{\alpha}, l}^{e}$ is a $K^{l}$-space if we define the corresponding $K^{l}$-action by setting

$$
\xi \circ\left(k_{1}, \ldots, k_{l}\right)\left(A_{1} \times \cdots \times A_{l}\right):=\xi\left(R_{k_{1}}^{-1} A_{1} \times \cdots \times R_{k_{l}}^{-1} A_{l}\right) .
$$

Proposition 3.8. Let $T$ be effective and have near $M S J_{l}$. If $T^{\alpha}$ is weakly mixing then $T^{\alpha}$ is l-fold near simple. Moreover,

$$
\Xi_{T^{\alpha}, l}^{e}=\left\{\xi^{\alpha} \circ h \mid \xi \in \Xi_{T, l}^{e} \text { with } \operatorname{Supp} \xi \subset\left\{T_{g} \mid g \in G\right\}^{l} \text { and } h \in K^{l}\right\} .
$$

Proof. Take any $\lambda \in J_{l}^{e}\left(T^{\alpha}\right)$. Denote by $\bar{\lambda}$ the projection of $\lambda$ onto $X^{l}$. Then $\bar{\lambda} \in J_{l}^{e}(T)$. Since $T$ has near $\mathrm{MSJ}_{l}, \bar{\lambda}=\mu_{\xi, P}$ for some measure $\xi \in \Xi_{T, l}^{e}$ with Supp $\xi \subset\left\{T_{g} \mid g \in G\right\}^{l}$ and a partition $P$ of $\{1, \ldots, l\}$. It was noticed above that the measure $\xi^{\alpha}$ is well defined and $\xi^{\alpha} \in \Xi_{T^{\alpha}, l}^{e}$. The weak mixing of $T^{\alpha}$ implies that $\left(\mu \times \lambda_{K}\right)_{\xi^{\alpha}, P} \in J_{l}^{e}\left(T_{\alpha}\right)$. The marginal of $\left(\mu \times \lambda_{K}\right)_{\xi^{\alpha}, P}$ onto $X^{l}$ is $\mu_{\xi, P}$. Hence by Lemma 3.7 , there exist $k_{1}, \ldots, k_{l} \in K$ with $\left(\mu \times \lambda_{K}\right)_{\xi^{\alpha}, P} \circ\left(R_{k_{1}} \times \cdots \times R_{k_{l}}\right)=\lambda$. It remains to notice that $\left(\mu \times \lambda_{K}\right)_{\xi^{\alpha}, P} \circ\left(R_{k_{1}} \times \cdots \times R_{k_{l}}\right)=\left(\mu \times \lambda_{K}\right)_{\xi^{\alpha} \circ\left(k_{1}, \ldots, k_{l}\right), P}$. 
Corollary 3.9 [dJR, Theorem 5.4]. Let $T$ be effective and have $M S J_{l}$. If $T^{\alpha}$ is weakly mixing then $T^{\alpha}$ l-fold simple. Moreover, $C\left(T^{\alpha}\right)$ is generated by $\left\{T_{g}^{\alpha} \mid g \in\right.$ $C(G)\}$ and $\left\{R_{k} \mid k \in K\right\}$.

\section{A tYPiCAL TRANSFORMATION IS NOT 2-FOLD NEAR SIMPLE}

This section is devoted entirely to a proof of the fact that the 2-fold near simple transformations form a meager subset in $\operatorname{Aut}_{0}(X, \mu)$ (Theorem 4.9).

Let $Y$ and $Z$ be two Polish spaces and let $f: Y \rightarrow Z$ be a continuous map. We denote by $\operatorname{LocDen}(f)$ the set of locally dense points in $Y$. Recall that a point $y \in Y$ is called locally dense (with respect to $f$ ) if each neighborhood of $y$ is dense in some neighborhood of $f(y)[\mathrm{Ki}]$.

Lemma 4.1. Let $\operatorname{LocDen}(f)$ be dense in $Y$. Then the following holds:

(i) For any non-meager subset $A \subset Y$, the image $f(A)$ is non-meager in $Z$.

(ii) If $Y^{\prime}$ is a non-meager $G_{\delta}$ in $Y$ then $\operatorname{LocDen}\left(f \uparrow Y^{\prime}\right) \neq \emptyset$. Hence if $Y^{\prime}$ is non-empty and open (or is a dense $\left.G_{\delta}\right)$ then $\operatorname{LocDen}\left(f \uparrow Y^{\prime}\right)$ is dense in $Y^{\prime}$.

Proof. (i) was shown in [Ki] and [Ag1].

(ii) If $\operatorname{LocDen}\left(f \uparrow Y^{\prime}\right)=\emptyset$ then for any $y \in Y^{\prime}$ there exists a neighborhood $U(y) \subset Y^{\prime}$ such that $f(U(y))$ is nowhere dense in $Z$. Since $Y^{\prime}$ is second countable in the induced topology, we can find a countable family $U\left(y_{n}\right), n \in \mathbb{N}$, which covers the whole $Y^{\prime}$. It follows that $f\left(Y^{\prime}\right)=\bigcup_{n} f\left(U\left(y_{n}\right)\right)$ is meager. This contradicts to (i). The second claim of (ii) follows from the first one and the following simple observations:

- each non-empty open subset in $Y^{\prime}$ is non-meager in $Y$,

- if $Y^{\prime \prime} \subset Y^{\prime}$ then $\operatorname{LocDen}\left(f \uparrow Y^{\prime \prime}\right) \subset \operatorname{LocDen}\left(f \uparrow Y^{\prime}\right)$. (Note that this fact is true even without the assumption on the denseness of LocDen $(f)$.)

We state the following statement about 'functorial' properties of LocDen $(f)$ without proof since it is obvious.

\section{Lemma 4.2.}

(i) If $\phi: Y \rightarrow Y$ is a homeomorphism pushing down to $Z$, i.e. $f \circ \phi=\psi \circ f$ for a homeomorphism $\psi$ of $Z$ then $\operatorname{LocDen}(f)$ is invariant under $\phi$.

(ii) More generally, let $Y^{\prime}$ be another Polish space and let $f^{\prime}: Y^{\prime} \rightarrow Z$ and $g: Y \rightarrow Y^{\prime}$ be continuous maps such that $f=f^{\prime} \circ g$. Then $g(\operatorname{LocDen}(f)) \subset$ $\operatorname{LocDen}\left(f^{\prime}\right)$.

Given a subset $A \subset Y \times Z$ and a point $z \in Z$, we denote by $A[z]$ the crosssection of $A$ over $z$, i.e. $A[z]:=\{y \in Y \mid(y, z) \in A\}$. Let $K$ be a compact second countable group. We denote by $\mathcal{M}(X, K)$ the group of measurable maps from $X$ to $K$. It is well known that $\mathcal{M}(X, K)$ is a Polish group when endowed with the topology of convergence in measure. Given $\phi \in \mathcal{M}(X, K)$, we denote by $T_{\phi} \in \operatorname{Aut}_{0}\left(X \times K, \mu \times \lambda_{K}\right)$ the skew product extension of $T$ via $\phi$, i.e. $T_{\phi}(x, k)=$ $(T x, \phi(x) k)$. The following well known facts will be crucial in the proof of the main results of this section.

Lemma 4.3. If $T$ be an ergodic transformation of $(X, \mathfrak{B}, \mu)$ then

(i) the subset $\left\{\phi \in \mathcal{M}(X, K) \mid T_{\phi}\right.$ is ergodic $\}$ is a dense $G_{\delta}$ in $\mathcal{M}(X, K)$; 
(ii) for any $\phi \in \mathcal{M}(X, K)$, the subset $\left\{a^{-1} \cdot \phi \cdot a \circ T \mid a \in \mathcal{M}(X, K)\right\}$-i.e. the cohomology class of $\phi-i s$ dense in $\mathcal{M}(X, K)$.

We introduce the following notation.

$$
\begin{aligned}
\mathcal{E} & :=\left\{T \in \operatorname{Aut}_{0}(X, \mu) \mid T \text { is ergodic }\right\}, \\
\Omega_{3} & :=\left\{S \in \operatorname{Aut}_{0}(X, \mu) \mid S^{3}=\mathrm{Id}\right\}, \\
\Omega_{3}^{*} & :=\left\{S \in \Omega_{3} \mid \mu(\{x \in X \mid S x=x\})=0\right\}, \\
\Omega_{\mathrm{com}} & :=\left\{(T, S) \in \operatorname{Aut}_{0}(X, \mu) \times \Omega_{3} \mid T S=S T\right\}, \\
\Omega_{\mathrm{com}}^{*} & \left.:=\Omega_{\mathrm{com}} \cap \operatorname{Aut}_{0}(X, \mu) \times \Omega_{3}^{*}\right), \\
\Omega_{\mathrm{fix}} & :=\left\{(T, S) \in \operatorname{Aut}_{0}(X, \mu) \times \Omega_{3} \mid T(\operatorname{Fix}(S))=\operatorname{Fix}(S)\right\}, \\
\Omega_{\mathrm{fix}}^{*} & :=\Omega_{\mathrm{fix}} \cap\left(\operatorname{Aut}_{0}(X, \mu) \times \Omega_{3}^{*}\right) .
\end{aligned}
$$

(We recall that $\operatorname{Fix}(S) \subset \mathfrak{B}$ denotes the sub- $\sigma$-algebra of subsets fixed by $S$.) All these spaces endowed with the corresponding induced topologies are Polish since:

$$
\begin{aligned}
& \mathcal{E} \text { is a dense } G_{\delta} \text { in } \operatorname{Aut}_{0}(X, \mu) ; \\
& \Omega_{3}^{*} \text { is a dense } G_{\delta} \text { in } \Omega_{3} \text { which, in turn, is closed in } \operatorname{Aut}_{0}(X, \mu) ; \\
& \Omega_{\text {com }} \text { and } \Omega_{\text {fix }} \text { are closed in } \operatorname{Aut}_{0}(X, \mu) \times \Omega_{3} .
\end{aligned}
$$

Let $\pi: \Omega_{\text {com }} \rightarrow \operatorname{Aut}_{0}(X, \mu)$ denote the marginal projection $(T, S) \mapsto T$.

Lemma 4.4. There exists $(T, S) \in \operatorname{LocDen}\left(\pi \uparrow \Omega_{\mathrm{com}}^{*}\right)$ such that $T$ is ergodic.

Proof. It was shown in [Ag1] that $\operatorname{LocDen}(\pi)$ is dense in $\Omega_{\mathrm{com}}$. By (4-1) and (4-2), the subsets $\pi^{-1}(\mathcal{E})$ and $\pi^{-1}(\mathcal{E}) \cap \Omega_{\text {com }}^{*}$ are $G_{\delta}$ in $\Omega_{\text {com }}$. If $\pi^{-1}(\mathcal{E})$ is not dense in $\Omega_{\text {com }}$ then $\pi^{-1}\left(\operatorname{Aut}_{0}(X, \mu) \backslash \mathcal{E}\right)$ is not meager. However this contradicts to Lemma 4.1(i) and (4-1). Notice that

$$
\pi^{-1}(\mathcal{E}) \subset \Omega_{\text {com }}^{*} \sqcup\left(\operatorname{Aut}_{0}(X, \mu) \times\{\operatorname{Id}\}\right) .
$$

It follows that $\pi^{-1}(\mathcal{E}) \cap \Omega_{\text {com }}^{*}$ is non-meager since it is dense in the non-empty open subset $\Omega_{\text {com }} \backslash\left(\operatorname{Aut}_{0}(X, \mu) \times\{\operatorname{Id}\}\right)$ of $\Omega_{\text {com }}$. Applying Lemma 4.1(ii), we conclude that

$$
\emptyset \neq \operatorname{LocDen}\left(\pi \uparrow\left(\pi^{-1}(\mathcal{E}) \cap \Omega_{\mathrm{com}}^{*}\right)\right) \subset \operatorname{LocDen}\left(\pi \uparrow \Omega_{\mathrm{com}}^{*}\right)
$$

Now we let $\mathcal{F}_{3}:=\left\{\operatorname{Fix}(S) \mid S \in \Omega_{3}^{*}\right\}$ and endow it with the quotient-Polishtopology with respect to the map $\Omega_{3}^{*} \ni S \mapsto \operatorname{Fix}(S) \in \mathcal{F}_{3}$. We also set

$$
\Omega_{\text {fix }}^{* *}:=\left\{(T, \mathfrak{F}) \in \operatorname{Aut}_{0}(X, \mu) \times \mathcal{F}_{3} \mid T(\mathfrak{F})=\mathfrak{F}\right\} .
$$

Clearly, $\Omega_{\text {fix }}^{* *}$ is a closed subset of $\operatorname{Aut}_{0}(X, \mu) \times \mathcal{F}_{3}$. Hence it is Polish. We fix a sub- $\sigma$-algebra $\mathfrak{F}_{0} \in \mathcal{F}_{3}$. Denote by $\operatorname{Aut}_{0}\left(\mathfrak{F}_{0}\right)$ the subgroup of those transformations of $X$ that preserve $\mathfrak{F}_{0}$ invariant.

Lemma 4.5. If $R \in \operatorname{Aut}_{0}\left(\mathfrak{F}_{0}\right)$ is ergodic then its conjugacy class in $\operatorname{Aut}_{0}\left(\mathfrak{F}_{0}\right)$ is dense.

Proof. Without loss of generality we may assume that there exists a standard probability space $\left(Y, \mathfrak{B}_{Y}, \nu\right)$ such that $(X, \mu)=\left(Y \times \mathbb{Z} / 3 \mathbb{Z}, \nu \times \lambda_{\mathbb{Z} / 3 \mathbb{Z}}\right)$ and $\mathfrak{F}_{0}=\mathfrak{B}_{Y} \otimes$ 
$\mathfrak{N}_{\mathbb{Z} / 3 \mathbb{Z}}$. Moreover, for each transformation $R \in \operatorname{Aut}_{0}\left(\mathfrak{F}_{0}\right)$, there exist $T \in \operatorname{Aut}_{0}(Y, \nu)$ and $\sigma \in \mathcal{M}\left(Y, \Sigma_{3}\right)$ such that

$$
R(y, i)=\widehat{T_{\sigma}}(y, i):=(T y, \sigma(y)(i)) .
$$

Furthermore, the map $\operatorname{Aut}_{0}\left(\mathfrak{F}_{0}\right) \ni \widehat{T_{\sigma}} \mapsto(T, \sigma) \in \operatorname{Aut}_{0}(Y, \nu) \ltimes \mathcal{M}\left(Y, \Sigma_{3}\right)$ is a topological group isomorphism. The multiplication law in this semidirect product is given by

$$
(T, \sigma) \cdot(Q, \tau):=(T Q, \sigma \circ Q \cdot \tau) .
$$

Clearly, if $R=\widehat{T_{\sigma}}$ then $T$ is ergodic. Now it remains to make use of Lemma 4.3(ii) and the classical fact that the conjugacy class of $T$ in $\operatorname{Aut}_{0}(Y, \nu)$ is dense.

Let $\pi^{\prime}: \Omega_{\mathrm{fix}}^{* *} \rightarrow \operatorname{Aut}_{0}(X, \mu)$ denote the marginal projection $(T, \mathfrak{F}) \mapsto T$.

Proposition 4.6. $\operatorname{LocDen}\left(\pi^{\prime}\right)$ is dense in $\Omega_{\mathrm{fix}}^{* *}$.

Proof. We first show that there exists $(T, \mathfrak{F}) \in \operatorname{LocDen}\left(\pi^{\prime}\right)$ such that $T$ is ergodic. Consider the following continuous maps

$$
\begin{aligned}
& \pi^{\prime \prime}: \Omega_{\mathrm{fix}}^{*} \ni(T, S) \mapsto T \in \mathcal{E} \text { and } \\
& f^{\prime}: \Omega_{\mathrm{fix}}^{*} \ni(T, S) \mapsto(T, \operatorname{Fix}(S)) \in \Omega_{\mathrm{fix}}^{* *}
\end{aligned}
$$

Since $\pi^{\prime \prime} \uparrow \Omega_{\text {com }}^{*}=\pi \uparrow \Omega_{\text {com }}^{*}$, it follows from Lemma 4.4 (see also the last phrase in the proof of Lemma 4.1) that $\operatorname{LocDen}\left(\pi^{\prime \prime}\right)$ contains a point $(T, S)$ with $T$ ergodic. Since $\pi^{\prime} \circ f^{\prime}=\pi^{\prime \prime}$, we derive from Lemma 4.2(ii) that $(T, \operatorname{Fix}(S)) \in \operatorname{LocDen}\left(\pi^{\prime}\right)$, as claimed.

Next we observe that $\Omega_{\mathrm{fix}}^{* *}$ is a topological $\operatorname{Aut}_{0}(X, \mu)$-space, if we set

$$
R \bullet(T, \mathfrak{F}):=\left(R T R^{-1}, R(\mathfrak{F})\right)
$$

for all $R \in \operatorname{Aut}_{0}(X, \mu)$ and $(T, \mathfrak{F}) \in \Omega_{\text {fix }}^{* *}$. Since $\pi^{\prime}$ is $\operatorname{Aut}_{0}(X, \mu)$-equivariant, it follows from Lemma 4.2(i) that the subset $\operatorname{LocDen}\left(\pi^{\prime}\right)$ is $\operatorname{Aut}_{0}(X, \mu)$-invariant. On the other hand, the other marginal projection $\Omega_{\text {fix }}^{* *} \ni(T, \mathfrak{F}) \mapsto \mathfrak{F} \in \mathcal{F}_{3}$ is also $\operatorname{Aut}_{0}(X, \mu)$-equivariant while $\mathcal{F}_{3}$ is just a single $\operatorname{Aut}_{0}(X, \mu)$-orbit. This clarifies the fiber structure of $\operatorname{LocDen}\left(\pi^{\prime}\right)$ : for any pair $\mathfrak{F}, \mathfrak{F}^{\prime} \in \mathcal{F}_{3}$, the cross-sections $\operatorname{LocDen}\left(\pi^{\prime}\right)[\mathfrak{F}]$ and $\operatorname{LocDen}\left(\pi^{\prime}\right)\left[\mathfrak{F}^{\prime}\right]$ are conjugate in $\operatorname{Aut}_{0}(X, \mu)$. To prove the proposition, it suffices to show that $\operatorname{LocDen}\left(\pi^{\prime}\right)\left[\mathfrak{F}_{0}\right]$ is dense in $\operatorname{Aut}_{0}\left(\mathfrak{F}_{0}\right)$. Since $\operatorname{LocDen}\left(\pi^{\prime}\right)\left[\mathfrak{F}_{0}\right]$ contains an ergodic transformation and is invariant under conjugation by any element of $\operatorname{Aut}_{0}\left(\mathfrak{F}_{0}\right)$, it remains to apply Lemma 4.5.

Proposition 4.7. There exists a dense $G_{\delta}$-subset $\Omega \subset \operatorname{Aut}_{0}\left(\mathfrak{F}_{0}\right)$ consisting of non2-fold near simple transformations.

Proof. Below we use the notation from the proof of Lemma 4.5. Let $\theta \in \Sigma_{3}$ denotes the flip, i.e. $\theta(i)=-i$ for all $i \in \mathbb{Z} / 3 \mathbb{Z}$. Since the map

$$
\operatorname{Aut}_{0}(Y, \nu) \ltimes \mathcal{M}\left(Y, \Sigma_{3}\right) \ni(T, \sigma) \mapsto T_{\sigma} \in \operatorname{Aut}_{0}\left(Y \times \Sigma_{3}, \nu \times \lambda_{\Sigma_{3}}\right)
$$

is continuous, it follows from (4-1) that the subset

$$
\Omega:=\left\{\widehat{T_{\sigma}} \mid T_{\sigma} \text { and } T_{\theta \sigma} \text { are both ergodic }\right\}
$$


is a $G_{\delta}$ in $\operatorname{Aut}_{0}\left(\mathfrak{F}_{0}\right)$. (Do not confuse the compact group extension $T_{\sigma}$ of $T$ with the isometric extension $\widehat{T_{\sigma}}$ of $T$.) In view of (4-1) and Lemma 4.3(i), we deduce from Kuratowski-Ulam theorem $[\mathrm{Ku}]$ that $\Omega$ is residual (= dense) in $\operatorname{Aut}_{0}\left(\mathfrak{F}_{0}\right)$.

It remains to show that each $\widehat{T_{\sigma}} \in \Omega$ is non-2-fold near simple. To this end we first notice that if $T_{\sigma}$ is ergodic then

$$
\left\{V \in C\left(\widehat{T_{\sigma}}\right) \mid V \uparrow \mathfrak{F}_{0}=\operatorname{Id}\right\}=\{\operatorname{Id}\}
$$

This fact follows, for instance, from [DaL, Proposition 2.5]. We now define two transformations $Q$ and $S$ of $(X, \mathfrak{B}, \mu)$ by setting

$$
Q(x, i):=(x,-i) \quad \text { and } \quad S(x, i):=(x, i+1) .
$$

Since $\widehat{T_{\sigma}}\left(\left\{x, S x, S^{2} x\right\}\right)=\left\{\widehat{T_{\sigma}} x, S \widehat{T_{\sigma}} x, S^{2} \widehat{T_{\sigma}} x\right\}$, it follows that $\widehat{T_{\sigma}}\left(\left\{S y, S^{2} y\right\}\right)=$ $\left\{S \widehat{T_{\sigma}} y, S^{2} \widehat{T_{\sigma}} y\right\}$. Therefore the measure

$$
\rho:=0.5\left(\mu_{S}+\mu_{S^{2}}\right)=0.5 \int_{X} \delta_{x} \times\left(\delta_{S x}+\delta_{S^{2} x}\right) d \mu(x)
$$

is a 2-fold self-joining of $\widehat{T_{\sigma}}$. If $\rho$ is not ergodic then $\rho=0.5\left(\rho_{1}+\rho_{2}\right)$ for some $\rho_{1}, \rho_{2} \in J_{2}\left(\widehat{T_{\sigma}}\right)$ with $\rho_{1} \perp \rho_{2}$. Since the marginal projections $(X \times X, \rho) \rightarrow(X, \mu)$ are 2-to-one and $\rho_{1} \perp \rho_{2}$, it follows that $\rho_{1}$ and $\rho_{2}$ are both one-to-one. Hence there are transformations $R_{1} \neq R_{2} \in C\left(\widehat{T_{\sigma}}\right)$ such that $\rho_{i}=\mu_{R_{i}}, i=1$, 2. Since the graph of $R_{i}$ is contained in the union of the graphs of $S$ and $S^{2}$, we obtain that $R_{i} \uparrow\left(\mathfrak{B}_{Y} \otimes \mathfrak{N}_{\mathbb{Z} / 3 \mathbb{Z}}\right)=\mathrm{Id}$. This contradicts to (4-4). Thus, $\rho \in J_{2}^{e}\left(\widehat{T_{\sigma}}\right)$. Since $\rho$ is not off-diagonal and $\rho \neq \mu \times \mu$, it follows that $\widehat{T_{\sigma}}$ is not 2 -fold simple. Hence if $\widehat{T_{\sigma}}$ is 2-fold near simple then there are transformations $V_{1} \neq V_{2} \in \operatorname{Aut}_{0}(X, \mu)$ such that $\rho=0.5\left(\nu_{V_{1}}+\nu_{V_{2}}\right)$, $\widehat{T_{\sigma}} V_{1}{\widehat{T_{\sigma}}}^{-1}=V_{2}$ and $\widehat{T_{\sigma}} V_{2}{\widehat{T_{\sigma}}}^{-1}=V_{1}$. We observe that $V_{i} x=S^{b_{i}(x)} x$ at a.a. $x$ for some measurable maps $b_{i}: X \rightarrow\{1,2\}$. Moreover, $\left\{b_{1}(x), b_{2}(x)\right\}=\{1,2\}$ and hence $b_{2}(x)=-b_{1}(x) \bmod 3$, i.e. $V_{2}=V_{1}^{-1}$. We also observe that $Q V_{1} Q^{-1}=V_{2}$. Hence $V_{1}$ commutes with $Q \widehat{T_{\sigma}}=\widehat{T_{\theta \sigma}}$. Since $V_{1} \uparrow$ $\left(\mathfrak{B}_{Y} \otimes \mathfrak{N}_{\mathbb{Z} / 3 \mathbb{Z}}\right)=\mathrm{Id}$, it follows from (4-3) and (4-4) that $V_{1}=\mathrm{Id}$, a contradiction.

Now we state a 0-1 law from $[\mathrm{GlK}]$.

Lemma 4.8. If an analytic subset of $\operatorname{Aut}_{0}(X, \mu)$ is $\operatorname{Aut}_{0}(X, \mu)$-invariant then it is either meager or residual.

Denote by $\mathcal{N} \subset \operatorname{Aut}_{0}(X, \mu)$ the subset of non-2-fold near simple transformations of $(X, \mathfrak{B}, \mu)$. It is clear that $\mathcal{N}$ is $\operatorname{Aut}_{0}(X, \mu)$-invariant. Now we will prove the main result of this section.

Theorem 4.9. $\mathcal{N}$ is residual.

Proof. Since the smallest $\operatorname{Aut}_{0}(X, \mu)$-invariant subset containing a given analytic subset is again analytic, it suffices to show that $\mathcal{N}$ contains a non-meager analytic subset (and apply Lemma 4.8). As we noted in the prof of Proposition 4.6, $\mathcal{F}_{3}$ is a homogeneous $\operatorname{Aut}_{0}(X, \mu)$-space homeomorphic to $\operatorname{Aut}_{0}(X, \mu) / \operatorname{Aut}_{0}\left(\mathfrak{F}_{0}\right)$. Hence there exists a Borel cross-section map $\mathcal{F}_{3} \ni \mathfrak{F} \mapsto R_{\mathfrak{F}} \in \operatorname{Aut}_{0}(X, \mu)$ such that $R_{\mathfrak{F}}\left(\mathfrak{F}_{0}\right)=\mathfrak{F}$ for all $\mathfrak{F}$. Hence this map is continuous on a dense $G_{\delta}$-subset $\mathcal{F}_{3}^{\prime} \subset \mathcal{F}_{3}$. 
Notice that the subset $\Omega^{\prime}:=\left\{(T, \mathfrak{F}) \mid \mathfrak{F} \in \mathcal{F}_{3}^{\prime}\right\}$ is a $G_{\delta}$ in $\Omega_{\text {fix }}^{* *}$. Moreover, it is residual (=dense) since the marginal projection $(T, \mathfrak{F}) \mapsto \mathfrak{F}$ is open. (The latter follows from the fact that this projection is equivariant and $\mathfrak{F}_{3}$ is a homogeneous $\operatorname{Aut}_{0}(X, \mu)$-space.) On the other hand, $\Omega^{\prime}$ is homeomorphic to the direct product $\operatorname{Aut}_{0}\left(\mathfrak{F}_{0}\right) \times \mathcal{F}_{3}^{\prime}$ via the map $\Phi:(T, \mathfrak{F}) \mapsto\left(R_{\mathfrak{F}}^{-1} T R_{\mathfrak{F}}, \mathfrak{F}\right)$. Now we deduce from Proposition 4.7 that $\Phi^{-1}\left(\Omega \times \mathfrak{F}_{3}^{\prime}\right)$ is a dense $G_{\delta}$ in $\Omega_{\text {fix }}^{* *}$ (the subset $\Omega$ was defined in Proposition 4.7). Hence by Lemma 4.1(i) and Proposition 4.6, $\pi^{\prime}\left(\Phi^{-1}\left(\Omega \times \mathfrak{F}_{3}^{\prime}\right)\right)$ is non-meager. It remains to notice that every transformation $T \in \pi^{\prime}\left(\Phi^{-1}\left(\Omega \times \mathfrak{F}_{3}^{\prime}\right)\right)$ is conjugate to some transformation from $\Omega$ (by the definition of $\pi^{\prime}$ and $\Phi$ ). Hence $T \in \mathcal{N}$ by Proposition 4.7 .

\section{NON-SIMPLE NEAR SIMPLE TRANSFORMATIONS}

Our purpose in this section is to construct transformations which are near simple but not 2 -fold simple.

Let $T$ be an ergodic transformation of $(X, \mathfrak{B}, \mu)$. The corresponding $\mathbb{Z}$-action $\mathbb{Z} \ni n \mapsto T^{n} \in \operatorname{Aut}_{0}(X, \mu)$ will be also denoted by $T$. Fix a prime $p>2$. Let $\mathcal{M}(X, \mathbb{Z} / p \mathbb{Z})$ stand for the group of measurable maps from $X$ to $\mathbb{Z} / p \mathbb{Z}$. As in the previous section, for each $\phi \in \mathcal{M}(X, \mathbb{Z} / p \mathbb{Z})$, we denote by $T_{\phi}$ the skew product extension of $T$ via $\phi$. We also define an 'adjoint to $T_{\phi}$ ' transformation $\widehat{T_{\phi}}$ of the same measure space $\left(X \times \mathbb{Z} / p \mathbb{Z}, \mu \times \lambda_{\mathbb{Z} / p \mathbb{Z}}\right)$ as follows:

$$
\widehat{T_{\phi}}(x, i)=(T x, \phi(x)-i) .
$$

It is easy to verify that ${\widehat{T_{\phi}}}^{2}=\left(T^{2}\right)_{\phi \circ T-\phi}$. Recall that a cocycle $\phi$ of $T$ is called ergodic if $T_{\phi}$ is ergodic. If the transformation $T^{2}$ is ergodic then for a residual subset of functions $\phi \in \mathcal{M}(X, \mathbb{Z} / p \mathbb{Z})$, the $T^{2}$-cocycle $\phi \times \phi \circ T: X \rightarrow(\mathbb{Z} / p \mathbb{Z})^{2}$ is ergodic [Da1]. Hence the $T^{2}$-cocycle $\phi \circ T-\phi: X \rightarrow \mathbb{Z} / p \mathbb{Z}$ is also ergodic. Thus we have proved the following: if $T^{2}$ is ergodic then ${\widehat{T_{\phi}}}^{2}$ (and hence $\widehat{T_{\phi}}$ ) is ergodic for a residual subset of $\phi \in \mathcal{M}(X, \mathbb{Z} / p \mathbb{Z})$.

Lemma 5.1. If $T$ is weakly mixing then the transformation ${\widehat{T_{\phi}}}^{2}$ (and hence $\widehat{T_{\phi}}$ ) is weakly mixing for a residual subset of $\phi \in \mathcal{M}(X, \mathbb{Z} / p \mathbb{Z})$.

Proof. Let $\phi$ belong to a residual subset of $\mathcal{M}(X, \mathbb{Z} / p \mathbb{Z})$ such that the $T^{2}$-cocycle $\phi \circ T-\phi$ is ergodic. If the transformation $T_{\phi \circ T-\phi}^{2}$ is not weakly mixing then there exists $c \in \mathbb{T} \backslash\{1\}$, a group character $\chi: \mathbb{Z} / p \mathbb{Z} \rightarrow \mathbb{T}$ and a map $f \in \mathcal{M}(X, \mathbb{T})$ such that $\chi \circ(\phi \circ T-\phi)=c \cdot f \cdot \overline{f \circ T^{2}}$. It follows that $c=g \circ T \cdot \bar{g}$, where $g:=\chi \circ \phi \cdot f \cdot f \circ T \in \mathcal{M}(X, \mathbb{T})$. However this contradicts to the weak mixing of $T$.

We need an auxiliary statement which follows from [dJR, Theorem 5.4 and Remark on p. 551] (cf. Corollary 3.9).

Lemma 5.2. Let a transformation $T$ be l-fold simple. If $K$ is a compact group and $\phi: X \rightarrow K$ a cocycle of $T$ such that

(i) $T_{\phi}$ is weakly mixing and

(ii) any transformation $S \in C(T)$ lifts to some $\widetilde{S} \in C\left(T_{\phi}\right)$

then $T_{\phi}$ is also l-fold simple and the group $C\left(T_{\phi}\right)$ is generated by all $\widetilde{S}$.

Now we state the main result of this section. 
Theorem 5.3. Let a transformation $T$ have $M S J_{l}$. Then for a residual subset of $\phi \in \mathcal{M}(X, \mathbb{Z} / p \mathbb{Z})$, the transformation $\widehat{T_{\phi}}$ is l-fold near simple but not 2-fold simple. Moreover, $C\left(\widehat{T_{\phi}}\right)=\left\{{\widehat{T_{\phi}}}^{n} \mid n \in \mathbb{Z}\right\}$.

Proof. Take any $\phi \in \mathcal{M}(X, \mathbb{Z} / p \mathbb{Z})$ with ${\widehat{T_{\phi}}}^{2}$ being weakly mixing. Such a $\phi$ exists by Lemma 5.1. Notice that $T^{2}$ is 2-fold simple and $C\left(T^{2}\right)=\left\{T^{n} \mid n \in \mathbb{Z}\right\}$ by Corollary 3.3. Since $T$ lifts to $\widehat{T_{\phi}} \in C\left({\widehat{T_{\phi}}}^{2}\right)$, we can apply Lemma 5.2 to deduce that ${\widehat{T_{\phi}}}^{2}$ is $l$-fold simple and the group $C\left({\widehat{T_{\phi}}}^{2}\right)$ is generated by $\widehat{T_{\phi}}$ and a transformation $S$ of $(X \times(\mathbb{Z} / p \mathbb{Z}), \widehat{\mu})$ given by $S(x, i)=(x, i+1)$, where $\widehat{\mu}:=\mu \times \lambda_{\mathbb{Z} / p \mathbb{Z}}$. Notice that $S^{p}=\mathrm{Id}, S^{j} \neq \mathrm{Id}$ for $0<j<p$ and $\widehat{T_{\phi}} S{\widehat{T_{\phi}}}^{-1}=S^{-1}$. Hence $\Xi_{{\widehat{T_{\phi}}}^{2}}^{e}=\left\{\delta_{{\widehat{T_{\phi}}}^{n} S^{j}} \mid\right.$ $n \in \mathbb{Z}, 0 \leq j<p\}$. Now we derive from Proposition 3.2 that

$$
\begin{aligned}
\Xi_{\widehat{T_{\phi}}}^{e} & =\left\{0.5\left(\delta_{{\widehat{T_{\phi}}}^{n} S^{j}}+\delta_{{\widehat{T_{\phi}}}^{n} S^{j}} \circ 1\right) \mid n \in \mathbb{Z}, 0 \leq j<p\right\} \\
& =\left\{0.5\left(\delta_{{\widehat{T_{\phi}}}^{n} S^{j}}+\delta_{{\widehat{T_{\phi}}}^{n} S^{-j}}\right) \mid n \in \mathbb{Z}, 0 \leq j<p / 2\right\} .
\end{aligned}
$$

Since $\Xi_{\widehat{T_{\phi}}}^{e}$ contains not only Dirac $\delta$-measures, the transformation $\widehat{T_{\phi}}$ is not 2 -fold simple.

Notice that $I\left(\widehat{T_{\phi}}\right)=\left\{{\widehat{T_{\phi}}}^{n} S^{j} \mid n \in \mathbb{Z}, 0 \leq j<p\right\}$. Hence $K:=\left\{S^{j} \mid 0 \leq j<p\right\}$ is the only compact submodule of $I\left(\widehat{T_{\phi}}\right)$. Hence by Theorem $2.6, \operatorname{Fix}(K)=\mathfrak{B}_{X} \otimes$ $\mathfrak{N}_{\mathbb{Z} / p \mathbb{Z}}$ is the only non-trivial factor of $\widehat{T_{\phi}}$. It is $p$-to-one. We notice also that for any self-joining $\nu \in J_{2}^{e}\left(\widehat{T_{\phi}}\right) \backslash\{\widehat{\mu} \times \widehat{\mu}\}$, the two marginal extensions $\left(\widehat{T_{\phi}} \times \widehat{T_{\phi}}, \nu\right) \rightarrow\left(\widehat{T_{\phi}}, \widehat{\mu}\right)$ are either one-to-one or 2-to-one. It follows that every ergodic joining of $\widehat{T_{\phi}}$ (except for the product $\widehat{\mu} \times \widehat{\mu}$ ) is isometric over the marginals. Hence $\widehat{T_{\phi}}$ is 2-fold QS if $l=2$ or $\widehat{T_{\phi}}$ is QS if $l>2$ by [Ry2].

We note that $\widehat{T_{\phi}}$ has a simple factor $\mathfrak{B}_{X} \otimes \mathfrak{N}_{\mathbb{Z} / 3 \mathbb{Z}}$. In the following section we will make use of a more elaborated technique to construct a near simple transformation without simple factors.

\section{A NEAR SIMPLE QS TRANSFORMATION WHICH IS DISJOINT WITH ALL SIMPLE MAPS}

In this section we construct a near simple QS transformation which is disjoint from any simple map. We also show that this transformation is not prime and that it has no prime factors (Theorem 6.9).

We start by reminding the $(C, F)$-construction of funny rank-one actions (see [Da2], [Da3], [DaS] and [dJ] for details). Let $G$ be a countable group. Given a finite subset $F \subset G$, we denote by $\lambda_{F}$ the probability equidistributed on $F$. Now let $\left(F_{n}\right)_{n \geq 0}$ and $\left(C_{n}\right)_{n \geq 1}$ be two sequences of finite subsets in $G$ such that the following are satisfied:

$$
\begin{aligned}
& F_{0}=\{0\}, \# C_{n}>1, \\
& F_{n} C_{n+1} \subset F_{n+1}, \\
& F_{n} c \cap F_{n} c^{\prime}=\emptyset \text { for all } c \neq c^{\prime} \in C_{n+1}, \\
& \lim _{n \rightarrow \infty} \frac{\# F_{n}}{\# C_{1} \cdots \# C_{n}}<\infty .
\end{aligned}
$$


We put $X_{n}:=F_{n} \times C_{n+1} \times C_{n+2} \times \cdots$ and define a map $i_{n}: X_{n} \rightarrow X_{n+1}$ by setting

$$
i_{n}\left(f_{n}, d_{n+1}, d_{n+2}, \ldots\right):=\left(f_{n} d_{n+1}, d_{n+2}, \ldots\right)
$$

In view of (6-1), $X_{n}$ endowed with the infinite product topology is a compact Cantor space. It follows from (6-2) and (6-3) that $i_{n}$ is well defined and it is a topological embedding of $X_{n}$ into $X_{n+1}$. Denote by $X$ the topological inductive limit of the sequence $\left(X_{n}, i_{n}\right)_{n=1}^{\infty}$. In the sequel we will suppress the canonical embedding maps and just write $X=\bigcup_{n \geq 0} X_{n}$ with $X_{0} \subset X_{1} \subset \cdots$. Clearly, $X$ is a locally compact Polish totally disconnected space without isolated points. We define a finite measure $\mu_{n}$ on $X_{n}$ by setting

$$
\mu_{n}:=\alpha_{n}\left(\lambda_{F_{n}} \times \lambda_{C_{n+1}} \times \lambda_{C_{n+2}} \times \cdots\right),
$$

where $\alpha_{n}$ is a positive coefficient such that

$$
\alpha_{0}:=1 \text { and } \alpha_{n+1}:=\alpha_{n} \frac{\# F_{n+1}}{\# F_{n} \# C_{n+1}}
$$

The latter 'matching' condition yields that $\mu_{n+1} \uparrow X_{n}=\mu_{n}$. Hence there exists a unique $\sigma$-finite measure $\mu$ on the standard Borel $\sigma$-algebra $\mathfrak{B}$ of $X$ generated by the topology such that $\mu \uparrow X_{n}=\mu_{n}$. In particular, $\mu\left(X_{n}\right)=\alpha_{n}$ for all $n \geq 0$. It is easy to check that $\mu(X)<\infty$ if and only if (6-4) holds. After a normalization (i.e. by an appropriate change of $\left.\alpha_{0}\right)$ we may assume that $\mu(X)=1$. Suppose also that the following is satisfied:

(6-5) for any $g \in G$, there exists $m \geq 0$ with $g F_{n} C_{n+1} \subset F_{n+1}$ for all $n \geq m$.

For such $n$, take any $x \in X_{n} \subset X$ and write the expansion $x=\left(f_{n}, c_{n+1}, c_{n+2}, \ldots\right)$ with $f_{n} \in F_{n}$ and $c_{n+i} \in C_{n+i}, i>0$. Then we let

$$
T_{g} x:=\left(g f_{n} c_{n+1}, c_{n+2}, \ldots\right) \in X_{n+1} \subset X
$$

It follows from (6-5) that $T_{g}$ is a well defined homeomorphism of $X$. Moreover, $T_{g} T_{g^{\prime}}=T_{g g^{\prime}}$, i.e. $T:=\left(T_{g}\right)_{g \in G}$ is a topological action of $G$ on $X$.

Definition 6.1. We call $(X, \mathfrak{B}, \mu, T)$ the $(C, F)$-action of $G$ associated to the sequence $\left(F_{n}, C_{n+1}\right)_{n=0}^{\infty}(\mathrm{cf} .[\mathrm{dJ}],[\mathrm{Da} 2],[\mathrm{Da} 3],[\mathrm{DaS}])$.

We list without proof several properties of $T$. They can be verified easily by the reader (see also [Da2] and $[\mathrm{DaS}]$ ).

- $T$ is a minimal uniquely ergodic (i.e. strictly ergodic) free action of $G$.

- Two points $x=\left(f_{n}, c_{n+1}, c_{n+2}, \ldots\right)$ and $x^{\prime}=\left(f_{n}^{\prime}, c_{n+1}^{\prime}, c_{n+2}^{\prime}, \ldots\right) \in X_{n}$ are $T$-orbit equivalent if and only if $c_{i}=c_{i}^{\prime}$ eventually (i.e. for all large enough $i$ ). Moreover, $x^{\prime}=T_{g} x$ if and only if

$$
g=\lim _{i \rightarrow \infty} f_{n}^{\prime} c_{n+1}^{\prime} \cdots c_{n+i}^{\prime} c_{n+i}^{-1} \cdots c_{n+1}^{-1} f_{n}^{-1} .
$$


For each $A \subset F_{n}$, we let $[A]_{n}:=\left\{x=\left(f_{n}, c_{n+1}, \ldots\right) \in X_{n} \mid f_{n} \in A\right\}$ and call it an $n$-cylinder. The following holds:

$$
\begin{aligned}
& {[A]_{n} \cap[B]_{n}=[A \cap B]_{n}, \text { and }[A]_{n} \cup[B]_{n}=[A \cup B]_{n},} \\
& {[A]_{n}=\bigsqcup_{c \in C_{n+1}}[A c]_{n+1},} \\
& T_{g}[A]_{n}=[g A]_{n} \text { if } g A \subset F_{n}, \\
& \mu\left([A c]_{n+1}\right)=\frac{1}{\# C_{n+1}} \mu\left([A]_{n}\right) \text { for any } c \in C_{n+1}, \\
& \mu\left([A]_{n}\right)=\mu\left(X_{n}\right) \lambda_{F_{n}}(A)
\end{aligned}
$$

Moreover, for each measurable subset $B \subset X$,

$$
\lim _{n \rightarrow \infty} \min _{A \subset F_{n}} \mu\left(B \triangle \bigsqcup_{a \in A} T_{a}[0]_{n}\right)=0 .
$$

This means that $T$ has funny rank one (see [Fe] for the case of $\mathbb{Z}$-actions and [So] for the general case).

Now we will construct a special $(C, F)$-action to obtain a transformation disjoint from all simple ones. Let $G_{n}:=\bigoplus_{i=1}^{n} \mathbb{Z} / 3 \mathbb{Z}$. From now on for every $n$, we fix an embedding $G_{n} \ni g \mapsto(0, g) \in G_{n+1}$. Then $G_{\infty}:=\bigcup_{n=1}^{\infty} G_{n}$ is isomorphic to $\bigoplus_{i=1}^{\infty} \mathbb{Z} / 3 \mathbb{Z}$. We will consider two infinite countable groups

$$
H:=G_{1} \times \mathbb{Z} \text { and } G:=G_{\infty} \rtimes \mathbb{Z} \text {. }
$$

Recall that the multiplication in $G$ is given by

$$
(h, n)(k, m):=\left(h+(-1)^{n} k, n+m\right) .
$$

Notice that

$$
(h, n)(k, m)(h, n)^{-1}=\left((-1)^{n} k+h-(-1)^{m} h, m\right) .
$$

It follows that $\mathrm{FC}(G)=G_{\infty} \times 2 \mathbb{Z}$ with $\# g^{*} \leq 2$ for every $g \in G$ (see Remark 2.5 for the definitions). Moreover, the center $C(G)$ of $G$ is $\{0\} \times 2 \mathbb{Z}$. Given $a>0$, we denote by $I[a]$ the symmetric interval $\{m \in \mathbb{Z}|| m \mid<a\}$. We also set $I_{+}[a]:=I[a] \cup\{a\}$. Let $\left(r_{n}\right)_{n=0}^{\infty}$ be an increasing sequence of positive integers such that

$$
\lim _{n \rightarrow \infty} n^{4} / r_{n}=0 \text {. }
$$

Below-just after Lemma 6.2 - one more restriction on the growth of $\left(r_{n}\right)_{n=0}^{\infty}$ will be imposed. We define recurrently two other sequences $\left(a_{n}\right)_{n=0}^{\infty}$ and $\left(\widetilde{a}_{n}\right)_{n=0}^{\infty}$ by setting

$$
a_{0}=\widetilde{a}_{0}=1, a_{n+1}:=\widetilde{a}_{n}\left(2 r_{n}-1\right), \widetilde{a}_{n+1}:=a_{n+1}+(2 n+1) \widetilde{a}_{n} .
$$

For each $n \in \mathbb{N}$, we let

$$
\begin{aligned}
& H_{n}:=G_{1} \times I\left[r_{n}\right], F_{n}:=G_{n} \times I_{+}\left[a_{n}\right], \widetilde{F}_{n}:=G_{n} \times I_{+}\left[\widetilde{a}_{n}\right] \text { and } \\
& S_{n}:=G_{n} \times I_{+}\left[(2 n-1) \widetilde{a}_{n-1}\right] .
\end{aligned}
$$


We also consider a homomorphism $\phi_{n}: H \rightarrow G_{n+1} \rtimes \mathbb{Z} \subset G$ given by

$$
\phi_{n}(i, t):=(i, \underbrace{0, \ldots, 0}_{n \text { times }}, 2 \widetilde{a}_{n} t)
$$

We then have

$$
\begin{gathered}
S_{n} \subset F_{n}, \quad F_{n} S_{n}=S_{n} F_{n} \subset \widetilde{F}_{n} \subset G, \\
F_{n+1}=\bigsqcup_{h \in H_{n}} \widetilde{F}_{n} \phi_{n}(h)=\bigsqcup_{h \in H_{n}} \phi_{n}(h) \widetilde{F}_{n} \text { and } \\
S_{n}=\bigsqcup_{h \in G_{1} \times I[n]} \widetilde{F}_{n-1} \phi_{n-1}(h) .
\end{gathered}
$$

Now fix a sequence $\epsilon_{n} \rightarrow 0$ as $n \rightarrow \infty$. For any two finite sets $A, B$ and a map $\phi: A \rightarrow B$, the probability $\frac{1}{\# A} \sum_{a \in A} \delta_{\phi(a)}$ on $B$ will be denoted by $\operatorname{dist}_{a \in A} \phi(a)$. Given two measures $\kappa, \rho$ on a finite set $B$, we let $\|\kappa-\rho\|_{1}:=\sum_{b \in B}|\kappa(b)-\rho(b)|$.

Lemma 6.2. If $r_{n}$ is sufficiently large then there exists a map $s_{n}: H_{n} \rightarrow S_{n}$ such that for any $\delta \geq n^{-2} r_{n}$,

$$
\left\|\operatorname{dist}_{t \in\{0\} \times I[\delta]}\left(s_{n}(h t), s_{n}\left(h^{\prime} t\right), s_{n}\left(h^{\prime \prime} t\right)\right)-\lambda_{S_{n}} \times \lambda_{S_{n}} \times \lambda_{S_{n}}\right\|_{1}<\epsilon_{n}
$$

whenever $h \neq h^{\prime} \neq h^{\prime \prime} \in H_{n}$ with $\left\{h, h^{\prime}, h^{\prime \prime}\right\} \cdot(\{0\} \times I[\delta]) \subset H_{n}$.

The notation $h \neq h^{\prime} \neq h^{\prime \prime}$ means that $h, h^{\prime}, h^{\prime \prime}$ are pairwise different. A 'twodimensional' version of this lemma (i.e. with $h$ and $h^{\prime}$ only and without $h^{\prime \prime}$ ) was established in $[\mathrm{dJ}]$. Only a slight modification of that proof is needed to prove the 'three-dimensional' Lemma 6.2 (cf. Lemma 2.3 from [Da3]). We leave this to the reader as an exercise.

From now on we will assume that $r_{n}$ is large so that the conclusion of Lemma 6.2 is satisfied. For every $n \in \mathbb{N}$, we fix a map $s_{n}$ whose existence is asserted in the lemma. Without loss of generality we may assume that the following boundary condition holds

$$
s_{n}\left(i, r_{n}-1\right)=s_{n}\left(i,-r_{n}+1\right)=0 \text { for all } i \in G_{1} \text {. }
$$

Now we can define a map $c_{n+1}: H_{n} \rightarrow F_{n+1}$ by setting $c_{n+1}(h):=s_{n}(h) \phi_{n}(h)$. We also put $C_{n+1}:=c_{n+1}\left(H_{n}\right)$. It is easy to derive from (6-8) and (6-9) that (6-1)-(6-3) are satisfied for the sequence $\left(F_{n}, C_{n+1}\right)_{n=0}^{\infty}$. Moreover,

$$
\frac{\# F_{n+1}}{\# F_{n} \# C_{n+1}}=\frac{2 a_{n+1}}{2 a_{n}\left(2 r_{n}-1\right)}=\frac{\widetilde{a}_{n}}{a_{n}}=1+\frac{(2 n-1) \widetilde{a}_{n-1}}{a_{n}}=1+\frac{2 n-1}{2 r_{n-1}-1} .
$$

From this and (6-7) we deduce that (6-4) holds. Moreover, (6-11) implies (6-5). Thus the conditions (6-1)-(6-5) are all satisfied for $\left(F_{n}, C_{n+1}\right)_{n=0}^{\infty}$. Hence we can consider the associated $(C, F)$-action $T$ of $G$ on a standard probability space $(X, \mathfrak{B}, \mu)$.

We state an auxiliary lemma. 
Lemma 6.3. Let $f=f^{\prime} \phi_{n-1}(h)$ with $f^{\prime} \in \widetilde{F}_{n-1}$ and $h \in H$.

(i) Then we have

$$
\begin{gathered}
\widetilde{F}_{n-1} \phi_{n-1}\left(h \cdot G_{1} \times I[n-1]\right) \subset f S_{n} \subset \widetilde{F}_{n-1} \phi_{n-1}\left(h \cdot G_{1} \times I[n+1]\right) \text { and hence } \\
\frac{\#\left(f S_{n} \backslash \widetilde{F}_{n-1} \phi_{n-1}\left(h \cdot G_{1} \times I[n-1]\right)\right)}{\# S_{n}} \leq \frac{4}{2 n-1} .
\end{gathered}
$$

(ii) If, in addition, $f S_{n} \subset F_{n}$ then

$$
\frac{\#\left(A C_{n} \cap f S_{n}\right)}{\# S_{n}}=\lambda_{F_{n-1}}(A) \pm \frac{10}{n}
$$

for any subset $A \subset F_{n-1}$.

Proof. (i) We have

$$
f S_{n}=f^{\prime} \phi_{n-1}(h) \widetilde{F}_{n-1} \phi_{n-1}\left(G_{1} \times I[n]\right)=f^{\prime} \widetilde{F}_{n-1} \phi_{n-1}\left(h \cdot G_{1} \times I[n]\right) .
$$

Since $\widetilde{F}_{n-1} \widetilde{F}_{n-1} \subset \bigsqcup_{|i| \leq 1} \widetilde{F}_{n-1} \phi_{n-1}(0, i)$, there exists a partition of $\widetilde{F}_{n-1}$ into subsets $A_{i},|i| \leq 1$, such that $f^{\prime} A_{i} \subset \widetilde{F}_{n-1} \phi_{n-1}(0, i)$ for any $i$. Therefore

$$
f S_{n}=\bigsqcup_{i} f^{\prime} A_{i} \phi_{n-1}(0,-i) \phi_{n-1}\left((0, i) \cdot h \cdot G_{1} \times I[n]\right) .
$$

It remains to notice that $\bigsqcup_{i} f^{\prime} A_{i} \phi_{n-1}(0,-i)=\widetilde{F}_{n-1}$.

(ii) If $f S_{n} \subset F_{n}$ then the subset $K:=h \cdot G_{1} \times I[n-1]$ is contained in $H_{n-1}$. Therefore

$$
\begin{aligned}
\frac{\#\left(A C_{n} \cap f S_{n}\right)}{\# S_{n}} & =\frac{\sum_{h \in H_{n-1}} \#\left(A s_{n-1}(h) \phi_{n-1}(h) \cap \widetilde{F}_{n-1} \phi_{n-1}(K)\right) \pm \frac{4}{2 n-1}}{\# S_{n}} \\
& =\sum_{k \in K} \frac{\#\left(A s_{n-1}(h)\right)}{\# S_{n}} \pm \frac{3}{n} \\
& =\frac{\# A}{\# F_{n-1}} \cdot \frac{\# F_{n-1}}{\# \widetilde{F}_{n-1}} \cdot \frac{\# K \# \widetilde{F}_{n-1}}{\# S_{n}} \pm \frac{3}{n} \\
& =\lambda_{F_{n-1}}(A)\left(1 \pm \frac{1}{n}\right) \frac{\# I[n-1]}{\# I[n]} \pm \frac{3}{n} . \quad \square
\end{aligned}
$$

To describe the 2-fold self-joinings of the transformation $T_{(0,1)}$ we first need to establish that $T_{(0,1)}$ is ergodic. We will show more:

Proposition 6.4. The transformation $T_{(0,2)}$ (and hence $T_{(0,1)}$ ) is weakly mixing.

Proof. Let $h_{0}:=(0,1) \in H$ and $g_{n}:=\phi_{n}\left(h_{0}\right)$. Since $g_{n}=(0,2)^{\widetilde{a}_{n}}$, it suffices to show that the sequence $\left(g_{n}\right)_{n=1}^{\infty}$ is mixing for $T$, i.e.

$$
\lim _{n \rightarrow \infty} \mu\left(T_{g_{n}} D \cap D^{\prime}\right)=\mu(D) \mu\left(D^{\prime}\right)
$$


for every pair of measurable subsets $D, D^{\prime} \subset X$. Take any $A, B \subset F_{n}$. Notice that $g_{n} \in C(G)$ for all $n \in \mathbb{N}$. Hence

$$
g_{n} A c_{n+1}(h)=A s_{n}(h) \phi_{n}\left(h_{0} h\right)=A s_{n}(h) s_{n}\left(h_{0} h\right)^{-1} c_{n+1}\left(h_{0} h\right)
$$

whenever $h, h_{0} h \in H_{n}$. We set $F_{n}^{\prime}:=F_{n} \cap F_{n} S_{n} S_{n}, A^{\prime}:=A \cap F_{n}^{\prime}, B^{\prime}:=B \cap F_{n}^{\prime}$, $H_{n}^{\prime}:=H_{n} \cap\left(h_{0}^{-1} H_{n}\right)$. Then

$$
\begin{aligned}
\mu\left(T_{g_{n}}[A]_{n} \cap[B]_{n}\right) & =\mu\left(T_{g_{n}}\left[A^{\prime}\right]_{n} \cap\left[B^{\prime}\right]_{n}\right) \pm 2 \mu\left(\left[F_{n} \backslash F_{n}^{\prime}\right]_{n}\right) \\
& =\sum_{h \in H_{n}} \mu\left(T_{g_{n}}\left[A^{\prime} c_{n+1}(h)\right]_{n+1} \cap\left[B^{\prime}\right]_{n}\right)+\bar{o}(1) \\
& =\sum_{h \in H_{n}^{\prime}} \mu\left(T_{g_{n}}\left[A^{\prime} c_{n+1}(h)\right]_{n+1} \cap\left[B^{\prime}\right]_{n}\right) \pm\left(1-\frac{\# H_{n}^{\prime}}{\# H_{n}}\right)+\bar{o}(1) \\
& =\sum_{h \in H_{n}^{\prime}} \mu\left(\left[A^{\prime} s_{n}(h) s_{n}\left(h_{0} h\right)^{-1} c_{n+1}\left(h_{0} h\right)\right]_{n+1} \cap\left[B^{\prime}\right]_{n}\right)+\bar{o}(1) \\
& =\sum_{h \in H_{n}^{\prime}} \mu\left(\left[\left(A^{\prime} s_{n}(h) s_{n}\left(h_{0} h\right)^{-1} \cap B^{\prime}\right) c_{n+1}\left(h_{0} h\right)\right]_{n+1}\right)+\bar{o}(1) \\
& =\frac{1}{\# H_{n}} \sum_{h \in H_{n}^{\prime}} \mu\left(\left[A^{\prime} s_{n}(h) s_{n}\left(h_{0} h\right)^{-1} \cap B^{\prime}\right]_{n}\right)+\bar{o}(1) \\
& =\frac{1}{\# H_{n}} \sum_{h \in H_{n}^{\prime}} \lambda_{F_{n}}\left(A^{\prime} s_{n}(h) s_{n}\left(h_{0} h\right)^{-1} \cap B^{\prime}\right) \mu\left(X_{n}\right)+\bar{o}(1) \\
& =\frac{1}{\# H_{n}^{\prime}} \sum_{h \in H_{n}^{\prime}} \lambda_{F_{n}}\left(A^{\prime} s_{n}(h) \cap B^{\prime} s_{n}\left(h_{0} h\right)\right)+\bar{o}(1) \\
& =\frac{1}{\# H_{n}^{\prime}} \sum_{h \in H_{n}^{\prime}} \lambda_{F_{n}}\left(A s_{n}(h) \cap B s_{n}\left(h_{0} h\right)\right)+\bar{o}(1)
\end{aligned}
$$

where $\bar{o}(1)$ means (here and below) a sequence that goes to 0 and that does not depend on the choice of $A, B \subset F_{n}$. Let $\xi_{n}:=\operatorname{dist}_{h \in H_{n}^{\prime}}\left(s_{n}(h), s_{n}\left(h_{0} h\right)\right)$. Notice that

$$
\xi_{n}=\frac{1}{3} \sum_{i \in G_{1}} \text { dist }_{-r_{n}<t<r_{n}-1}\left(s_{n}(i, t), s_{n}(i, t+1)\right) .
$$

It follows from Lemma 6.2 that $\left\|\xi_{n}-\lambda_{S_{n}} \times \lambda_{S_{n}}\right\|_{1}<\epsilon_{n}$. We define a function $f: S_{n} \times S_{n} \rightarrow \mathbb{R}$ by setting $f(v, w):=\lambda_{F_{n}}(A v \cap B w)$. Then

$$
\frac{1}{\# H_{n}^{\prime}} \sum_{h \in H_{n}^{\prime}} \lambda_{F_{n}}\left(A s_{n}(h) \cap B s_{n}\left(h_{0} h\right)\right)=\int f d \xi_{n}=\int f d\left(\lambda_{S_{n}} \times \lambda_{S_{n}}\right) \pm \epsilon_{n} .
$$

Thus we obtain

$$
\mu\left(T_{g_{n}}[A]_{n} \cap[B]_{n}\right)=\int_{S_{n} \times S_{n}} \lambda_{F_{n}}(A v \cap B w) d \lambda_{S_{n}}(v) d \lambda_{S_{n}}(w)+\bar{o}(1) .
$$

Now we take $A:=A^{*} C_{n}$ and $B:=B^{*} C_{n}$ for some subsets $A^{*}, B^{*} \subset F_{n-1}$. Then the integral in the righthand side of (6-12) equals to the sum

$$
\sum_{a \in A^{*}} \sum_{b \in B^{*}} \sum_{h, h^{\prime} \in H_{n-1}} \frac{\#\left(a c_{n}(h) S_{n} \cap b c_{n}\left(h^{\prime}\right) S_{n} \cap F_{n}\right)}{\left(\# S_{n}\right)^{2} \# F_{n}} .
$$


It follows from the definition of $c_{n}$ and Lemma 6.3(i) that

$a c_{n}(h) S_{n} \cap b c_{n}\left(h^{\prime}\right) S_{n} \subset \widetilde{F}_{n-1} \phi_{n-1}\left(h \cdot G_{1} \times I[n+1]\right) \cap \widetilde{F}_{n-1} \phi_{n-1}\left(h^{\prime} \cdot G_{1} \times I[n+1]\right)$.

Hence $a c_{n}(h) S_{n} \cap b c_{n}\left(h^{\prime}\right) S_{n} \neq \emptyset$ only if $h^{\prime} h^{-1} \in G_{1} \times I[2 n+1]$. If the latter is satisfied we say that $h$ and $h^{\prime}$ are partners. Denote by $P(h)$ the set of partners of $h$ from $H_{n-1}$. Clearly, $\# P(h) \leq 12 n+3$. Therefore we deduce from (6-12), (6-13) and Lemma 6.3(i) that

$$
\begin{aligned}
\mu\left(T_{g_{n}}\left[A^{*}\right]_{n-1} \cap\left[B^{*}\right]_{n-1}\right) & \\
& =\sum_{a \in A^{*}} \sum_{b \in B^{*}} \sum_{h \in H_{n-1}} \sum_{h^{\prime} \in P(h)} \frac{\#\left(c_{n}(h) S_{n} \cap c_{n}\left(h^{\prime}\right) S_{n} \cap F_{n}\right) \pm \frac{4 \# S_{n}}{2 n-1}}{\left(\# S_{n}\right)^{2} \# F_{n}}+\bar{o}(1) \\
& =\frac{\# A^{*} \# B^{*}}{\left(\# F_{n-1}\right)^{2}} \cdot \theta_{n} \pm \frac{\left(\# F_{n-1}\right)^{2} \# H_{n-1} \cdot(12 n+3) \cdot 4 \# S_{n}}{\left(\# S_{n}\right)^{2} \# F_{n} \cdot(2 n-1)}+\bar{o}(1) \\
& =\lambda_{F_{n-1}}\left(A^{*}\right) \lambda_{F_{n-1}}\left(B^{*}\right) \theta_{n} \pm 7 \frac{\# F_{n-1}}{\# S_{n}}+\bar{o}(1),
\end{aligned}
$$

where $\theta_{n}$ is a positive number. Substituting $A^{*}=B^{*}=F_{n-1}$ and passing to the limit, we obtain that $\theta_{n} \rightarrow 1$ as $n \rightarrow \infty$. Hence

$$
\mu\left(T_{g_{n}}\left[A^{*}\right]_{n-1} \cap\left[B^{*}\right]_{n-1}\right)=\mu\left(\left[A^{*}\right]_{n-1}\right) \mu\left(\left[B^{*}\right]_{n-1}\right)+\bar{o}(1) .
$$

Since $\bar{o}(1)$ does not depend on the choice of $A^{*}$ and $B^{*}$ inside $F_{n-1}$, it follows from (6-6) and (6-14) that $\left(g_{n}\right)_{n=1}^{\infty}$ is mixing for $T$.

Remark 6.5. It is possible to show that $T_{(0,1)}$ is mixing. However it is not our purpose in this paper.

Our next task is to describe all ergodic 2-fold self-joinings of $T_{(0,1)}$. Given $(g, i) \in$ $G$, we let $(g, i)^{*}:=(0,1)(g, i)(0,1)^{-1}=(-g, i)$.

Theorem 6.6. $J_{2}^{e}\left(T_{(0,1)}\right)=\left\{\frac{1}{2}\left(\mu_{T_{k}}+\mu_{T_{k^{*}}}\right) \mid k \in G\right\} \cup\{\mu \times \mu\}$.

Proof. Take any joining $\nu \in J_{2}^{e}\left(T_{(0,1)}\right)$. Let $I_{n}:=I\left[n^{-2} a_{n}\right], J_{n}:=I\left[n^{-2} r_{n}\right]$ and $\Phi_{n}:=I_{n}+2 \widetilde{a}_{n} J_{n}$. We first notice that $\left(\Phi_{n}\right)_{n=1}^{\infty}$ is a Følner sequence in $\mathbb{Z}$. Since

$$
\frac{a_{n}}{n^{2}}+\frac{2 \widetilde{a}_{n} r_{n}}{n^{2}}<\frac{\widetilde{a}_{n}\left(2 r_{n}+1\right)}{n^{2}}<\frac{2 a_{n+1}}{(n+1)^{2}}
$$

it follows that $\Phi_{n} \subset I_{n+1}+I_{n+1}$ and hence $\bigcup_{m=1}^{n} \Phi_{m} \subset I_{n+1}+I_{n+1}$. This implies that

$$
\#\left(\Phi_{n+1}-\bigcup_{m=1}^{n} \Phi_{m}\right) \leq 3 \# \Phi_{n+1} \text { for every } n \in \mathbb{N}
$$

i.e. Shulman's condition [Li] is satisfied for $\left(\Phi_{n}\right)_{n=1}^{\infty}$. By [Li], the pointwise ergodic theorem holds along $\left(\Phi_{n}\right)_{n=1}^{\infty}$ for any ergodic transformation. Hence

$$
\frac{1}{\# \Phi_{n}} \sum_{i \in \Phi_{n}} \chi_{D}\left(T_{(0, i)} x\right) \chi_{D^{\prime}}\left(T_{(0, i)} x^{\prime}\right) \rightarrow \nu\left(D \times D^{\prime}\right)
$$


as $n \rightarrow \infty$ at $\nu$-a.a. $\left(x, x^{\prime}\right) \in X \times X$ for all cylinders $D, D^{\prime} \subset X$. We call such $\left(x, x^{\prime}\right)$ a generic point for $\left(T_{(0,1)} \times T_{(0,1)}, \nu\right)$. Fix one of them. Then $x, x^{\prime} \in X_{n}$ for all sufficiently large $n$ and we have the following expansions

$$
\begin{aligned}
x & =\left(f_{n}, c_{n+1}\left(h_{n}\right), c_{n+2}\left(h_{n+1}\right), \ldots,\right), \\
x^{\prime} & =\left(f_{n}^{\prime}, c_{n+1}\left(h_{n}^{\prime}\right), c_{n+2}\left(h_{n+1}^{\prime}\right), \ldots,\right)
\end{aligned}
$$

with $f_{n}, f_{n}^{\prime} \in F_{n}$ and $h_{i}, h_{i}^{\prime} \in H_{i}, i>n$. We let $H_{n}^{-}=G_{1} \times I\left[\left(1-n^{-2}\right) r_{n}\right] \subset H_{n}$. Then $\# H_{n}^{-} / \# H_{n} \geq 1-n^{-2}$. Since the marginals of $\nu$ are both equal to $\mu$, by Borel-Cantelli lemma we may assume without loss of generality that $h_{n}, h_{n}^{\prime} \in H_{n}^{-}$ for all sufficiently large $n$. This implies, in turn, that

$$
f_{n+1}=f_{n} c_{n+1}\left(h_{n}\right) \in \widetilde{F}_{n} \phi_{n}\left(H_{n}^{-}\right) \subset G_{n+1} \times I_{+}\left[\left(2 r_{n}\left(1-n^{-2}\right)-1\right) \widetilde{a}_{n}\right],
$$

and, similarly, $f_{n+1}^{\prime} \in G_{n+1} \times I_{+}\left[\left(2 r_{n}\left(1-n^{-2}\right)-1\right) \widetilde{a}_{n}\right]$. Notice that given $g \in \Phi_{n}$, we have $(0, g)=(0, b) \phi_{n}(0, t)$ for some uniquely determined $b \in I_{n}$ and $t \in J_{n}$. Moreover, $\phi_{n}(0, t) \in C(G)$. Furthermore, $(0, t) h_{n} \in H_{n}$. We also claim that

$$
(0, b) f_{n} S_{n} S_{n} \subset F_{n}
$$

if $n$ is large enough. To verify this, it suffices to show that

$$
\begin{aligned}
& \frac{a_{n}}{n^{2}}+2 r_{n-1}\left(1-\frac{1}{(n-1)^{2}}\right) \widetilde{a}_{n-1}+4 n \widetilde{a}_{n-1}<a_{n}, \text { i.e. } \\
& 2 r_{n-1}\left(1-\frac{1}{(n-1)^{2}}\right)+4 n<\left(2 r_{n-1}-1\right)\left(1-\frac{1}{n^{2}}\right) .
\end{aligned}
$$

The latter inequality, in turn, is equivalent to

$$
1+4 n-\frac{1}{n^{2}}<2 r_{n-1}\left(\frac{1}{(n-1)^{2}}-\frac{1}{n^{2}}\right),
$$

which follows from (6-7). Hence

$$
\begin{aligned}
& (0, g) f_{n} s_{n}\left(h_{n}\right) \phi_{n}\left(h_{n}\right)=d c_{n+1}\left((0, t) h_{n}\right) \text { and } \\
& (0, g) f_{n}^{\prime} s_{n}\left(h_{n}^{\prime}\right) \phi_{n}\left(h_{n}^{\prime}\right)=d^{\prime} c_{n+1}\left((0, t) h_{n}^{\prime}\right),
\end{aligned}
$$

where $d:=(0, b) f_{n} s_{n}\left(h_{n}\right) s_{n}\left((0, t) h_{n}\right)^{-1}$ and $d^{\prime}:=(0, b) f_{n}^{\prime} s_{n}\left(h_{n}^{\prime}\right) s_{n}\left((0, t) h_{n}^{\prime}\right)^{-1}$ belong to $F_{n}$ by (6-16). Now take any $B, B^{\prime} \subset F_{n-1}$ and set $A:=B C_{n} \subset F_{n}$ and $A^{\prime}:=B^{\prime} C_{n} \subset F_{n}$. We have

$$
\begin{aligned}
\#\{g & \left.\in \Phi_{n} \mid\left(T_{(0, g)} x, T_{(0, g)} x^{\prime}\right) \in[A]_{n} \times\left[A^{\prime}\right]_{n}\right\} \\
\# \Phi_{n} & \frac{1}{\# I_{n}} \sum_{b \in I_{n}} \frac{\#\left\{t \in J_{n} \mid d \in A, d^{\prime} \in A^{\prime}\right\}}{\# J_{n}} \\
& =\frac{1}{\# I_{n}} \sum_{b \in I_{n}} \xi_{n}\left(A^{-1}(0, b) f_{n} s_{n}\left(h_{n}\right) \times A^{\prime-1}(0, b) f_{n}^{\prime} s_{n}\left(h_{n}^{\prime}\right)\right),
\end{aligned}
$$


where $\xi_{n}:=\operatorname{dist}_{t \in J_{n}}\left(s_{n}\left((0, t) h_{n}\right), s_{n}\left((0, t) h_{n}^{\prime}\right)\right)$.

We consider separately two cases. Suppose first that $h_{n}=h_{n}^{\prime}$ for all $n$ greater than some $N$. Then it is easy to deduce from Lemma 6.2 that $\left\|\xi_{n}-\Delta\right\|_{1}<\epsilon_{n}$, where $\Delta$ is the probability equidistributed on the diagonal of $S_{n} \times S_{n}$. Moreover, $f_{n} f_{n}^{\prime-1}=f_{N} f_{N}^{\prime-1}=: k$ for all $n>N$. Then $(0, b) k(0, b)^{-1}=k$ or $(0, b) k(0, b)^{-1}=$ $k^{*}$ if $b \in I_{n}$ is even or odd respectively. This yields

$$
\begin{aligned}
\frac{1}{\# I_{n}} \sum_{b \in I_{n}} \xi_{n}\left(A^{-1}(0, b) f_{n} s_{n}\left(h_{n}\right) \times A^{\prime-1}(0, b) f_{n}^{\prime} s_{n}\left(h_{n}^{\prime}\right)\right) \\
=\frac{1}{\# I_{n}} \sum_{b \in I_{n}} \lambda_{S_{n}}\left(A^{-1}(0, b) f_{n} s_{n}\left(h_{n}\right) \cap A^{\prime-1}(0, b) f_{n}^{\prime} s_{n}\left(h_{n}\right)\right) \pm \epsilon_{n} \\
=\frac{1}{\# I_{n}} \sum_{b \in I_{n}} \frac{\#\left(A \cap(0, b) f_{N} f_{N}^{\prime-1}(0, b)^{-1} A^{\prime} \cap(0, b) f_{n} s_{n}\left(h_{n}\right) S_{n}\right)}{\# S_{n}} \pm \epsilon_{n} \\
=\frac{1}{\# I_{n}} \sum_{2 b \in I_{n}} \frac{\#\left(A \cap k A^{\prime} \cap(0,2 b) f_{n} s_{n}\left(h_{n}\right) S_{n}\right)}{\# S_{n}} \\
\quad+\frac{1}{\# I_{n}} \sum_{2 b+1 \in I_{n}} \frac{\#\left(A \cap k^{*} A^{\prime} \cap(0,2 b+1) f_{n} s_{n}\left(h_{n}\right) S_{n}\right)}{\# S_{n}}+\bar{o}(1) .
\end{aligned}
$$

Notice that $(0,2 b) f_{n} s_{n}\left(h_{n}\right) S_{n} \subset F_{n}$ by (6-16). Since $k \in F_{N} F_{N}^{-1}$, it follows that

$$
\max _{D \subset F_{n-1}} \frac{\#\left(D \backslash k^{-1} F_{n-1}\right)}{\# F_{n-1}}=\bar{o}(1)
$$

We now set $\widetilde{B}:=B^{\prime} \cap k^{-1} F_{n-1}$. Then Lemma 6.3 (ii) yields

$$
\begin{aligned}
& \frac{\#\left(B C_{n} \cap k B^{\prime} C_{n} \cap(0,2 b) f_{n} s_{n}\left(h_{n}\right) S_{n}\right)}{\# S_{n}} \\
& =\frac{\#\left((B \cap k \widetilde{B}) C_{n} \cap(0,2 b) f_{n} s_{n}\left(h_{n}\right) S_{n}\right)}{\# S_{n}}+\bar{o}(1) \\
& =\lambda_{F_{n-1}}(B \cap k \widetilde{B})+\bar{o}(1) \\
& =\frac{\mu\left([B \cap k \widetilde{B}]_{n-1}\right)}{\mu\left(X_{n-1}\right)}+\bar{o}(1) \\
& =\mu\left([B]_{n-1} \cap T_{k}\left[B^{\prime}\right]_{n-1}\right)+\bar{o}(1) .
\end{aligned}
$$

In a similar way,

$$
\frac{\#\left(B C_{n} \cap k^{*} B^{\prime} C_{n} \cap(0,2 b+1) f_{n} s_{n}\left(h_{n}\right) S_{n}\right)}{\# S_{n}}=\mu\left([B]_{n-1} \cap T_{k}\left[B^{\prime}\right]_{n-1}\right)+\bar{o}(1) .
$$

Therefore it follows from (6-15) that

$$
\nu\left([B]_{n-1} \times\left[B^{\prime}\right]_{n-1}\right)=\frac{1}{2}\left(\mu_{T_{k}}\left([B]_{n-1} \times\left[B^{\prime}\right]_{n-1}\right)+\mu_{T_{k^{*}}}\left([B]_{n-1} \times\left[B^{\prime}\right]_{n-1}\right)+\bar{o}(1) .\right.
$$

Then we deduce from (6-6) that $\nu=\frac{1}{2}\left(\mu_{T_{k}}+\mu_{T_{k^{*}}}\right)$. 
Now consider the second case, where $h_{n} \neq h_{n}^{\prime}$ for infinitely many, say bad $n$. It follows from Lemma 6.2 that $\left\|\xi_{n}-\lambda_{S_{n}} \times \lambda_{S_{n}}\right\|<\epsilon_{n}$ for all such $n$. Hence

$$
\begin{aligned}
& \frac{1}{\# I_{n}} \sum_{b \in I_{n}} \xi_{n}\left(A^{-1}(0, b) f_{n} s_{n}\left(h_{n}\right) \times A^{\prime-1}(0, b) f_{n}^{\prime} s_{n}\left(h_{n}^{\prime}\right)\right) \\
& \quad=\frac{1}{\# I_{n}} \sum_{b \in I_{n}} \lambda_{S_{n}}\left(A^{-1}(0, b) f_{n} s_{n}\left(h_{n}\right)\right) \lambda_{S_{n}}\left(A^{\prime-1}(0, b) f_{n}^{\prime} s_{n}\left(h_{n}^{\prime}\right)\right) \pm \epsilon_{n}
\end{aligned}
$$

Now we derive from Lemma 6.3(ii) and (6-16) that

$$
\lambda_{S_{n}}\left(A^{-1}(0, b) f_{n} s_{n}\left(h_{n}\right)\right)=\frac{\#\left(A \cap(0, b) f_{n} s_{n}\left(h_{n}\right) S_{n}\right)}{\# S_{n}}=\lambda_{F_{n-1}}(B) \pm \frac{10}{n}
$$

and, in a similar way, $\lambda_{S_{n}}\left(A^{\prime-1}(0, b) f_{n}^{\prime} s_{n}\left(h_{n}^{\prime}\right)\right)=\lambda_{F_{n-1}}\left(B^{\prime}\right) \pm \frac{10}{n}$. Hence

$$
\nu\left([B]_{n-1} \times\left[B^{\prime}\right]_{n-1}\right)=\mu\left([B]_{n-1}\right) \mu\left(\left[B^{\prime}\right]_{n-1}\right)+\bar{o}(1)
$$

provided that $n$ is bad. It remains to note that (6-6) holds along any subsequence, in particular along the subsequence of bad $n$. Hence $\nu=\mu \times \mu$.

Proposition 6.7. $T_{(0,1)}$ is 3-fold PID.

Sketch of the proof. Let a joining $\nu \in J_{3}^{e}\left(T_{(0,1)}\right)$ be pairwise independent. We need to show that $\nu=\mu \times \mu \times \mu$. To this end we will follow the same road as in the proof of Theorem 6.6. Fix a generic point $\left(x, x^{\prime}, x^{\prime \prime}\right)$ for $\left(T_{(0,1)} \times T_{(0,1)} \times T_{(0,1)}, \nu\right)$. Then $x, x^{\prime}, x^{\prime \prime} \in X_{n}$ for all sufficiently large $n$. Without loss of generality we may assume that

$$
\begin{aligned}
x & =\left(f_{n}, c_{n+1}\left(h_{n}\right), c_{n+2}\left(h_{n+1}\right), \ldots,\right), \\
x^{\prime} & =\left(f_{n}^{\prime}, c_{n+1}\left(h_{n}^{\prime}\right), c_{n+2}\left(h_{n+1}^{\prime}\right), \ldots,\right), \\
x^{\prime \prime} & =\left(f_{n}^{\prime \prime}, c_{n+1}\left(h_{n}^{\prime \prime}\right), c_{n+2}\left(h_{n+1}^{\prime \prime}\right), \ldots,\right)
\end{aligned}
$$

with $f_{n}, f_{n}^{\prime}, f_{n}^{\prime \prime} \in F_{n}$ and $h_{i}, h_{i}^{\prime}, h_{i}^{\prime \prime} \in H_{i}^{-}, i>n$. Now take any $B, B^{\prime}, B^{\prime \prime} \subset F_{n-1}$ and set $A:=B C_{n}, A^{\prime}:=B^{\prime} C_{n}, A^{\prime \prime}:=B^{\prime \prime} C_{n}$. Then as in the proof of Theorem 6.5 we have

$$
\begin{aligned}
& \frac{\#\left\{g \in \Phi_{n} \mid\left(T_{(0, g)} x, T_{(0, g)} x^{\prime}, T_{(0, g)} x^{\prime \prime}\right) \in[A]_{n} \times\left[A^{\prime}\right]_{n} \times\left[A^{\prime \prime}\right]_{n}\right\}}{\# \Phi_{n}} \\
& =\frac{1}{\# I_{n}} \sum_{b \in I_{n}} \xi_{n}\left(A^{-1} d \times A^{\prime-1} d^{\prime} \times A^{\prime \prime-1} d^{\prime \prime}\right),
\end{aligned}
$$

where $d:=(0, b) f_{n} s_{n}\left(h_{n}\right), d^{\prime}:=(0, b) f_{n}^{\prime} s_{n}\left(h_{n}^{\prime}\right), d^{\prime \prime}:=(0, b) f_{n}^{\prime \prime} s_{n}\left(h_{n}^{\prime \prime}\right)$ and $\xi_{n}:=$ $\operatorname{dist}_{t \in J_{n}}\left(s_{n}\left((0, t) h_{n}\right), s_{n}\left((0, t) h_{n}^{\prime}\right), s_{n}\left((0, t) h_{n}^{\prime \prime}\right)\right)$. Next, given $m>0$,

$$
\begin{aligned}
\frac{\mu \times \mu\left(\left\{\left(y, y^{\prime}\right) \in X_{m} \times X_{m} \mid y_{i} \neq y_{i}^{\prime} \text { for all } i>m\right\}\right)}{\mu\left(X_{m}\right) \mu\left(X_{m}\right)} & =\prod_{i>m} \frac{\# C_{i}^{2}-\# C_{i}}{\# C_{i}} \\
& =\prod_{i>m}\left(1-\frac{1}{\# H_{i}}\right)>0
\end{aligned}
$$


where $\left(y_{i}\right)_{i \geq m}$ and $\left(y_{i}^{\prime}\right)_{i \geq m}$ are the 'coordinates' of $y$ and $y^{\prime} \in X_{m}=F_{m} \times C_{m+1} \times$ $C_{m+2} \times \cdots$ respectively. Since $\nu$-a.e. point is generic for $T_{(0,1)} \times T_{(0,1)} \times T_{(0,1)}$ and $\nu$ is pairwise independent, we can select the generic point $\left(x, x^{\prime}, x^{\prime \prime}\right)$ in such a way that $h_{i} \neq h_{i}^{\prime} \neq h_{i}^{\prime \prime}$ for all $i \geq n$ whenever $n$ is large enough. Therefore it follows from Lemma 6.2 that $\left\|\xi_{i}-\lambda_{S_{i}} \times \lambda_{S_{i}} \times \lambda_{S_{i}}\right\|<\epsilon_{i}$ for all $i \geq n$. Now we derive from (6-17) and Lemma 6.3 that

$$
\nu\left([B]_{n-1} \times\left[B^{\prime}\right]_{n-1} \times\left[B^{\prime \prime}\right]_{n-1}\right)=\mu\left([B]_{n-1}\right) \mu\left(\left[B^{\prime}\right]_{n-1}\right) \mu\left(\left[B^{\prime \prime}\right]_{n-1}\right)+\bar{o}(1)
$$

This implies that $\nu=\mu \times \mu \times \mu$.

The following important result generalizing [dJR, Theorem 4.2, Corollary 4.3] and [Th1, Theorem 6, Corollary 4] was established in [dJL2, Proposition 7].

Lemma 6.8. Let $R$ and $S$ be weakly mixing transformations. Assume that $R$ is $D S$ and $S$ is simple. Then $R$ and $S$ are disjoint if and only if they have no non-trivial common factors.

Now we state the main results of this section.

\section{Theorem 6.9.}

(i) $T_{(0,2)}$ is simple and $C\left(T_{(0,2)}\right)=\left\{T_{g} \mid g \in G\right\}$.

(ii) $T_{(0,1)}$ is near simple, $Q S$ but not 2-fold simple. Moreover, $I\left(T_{(0,1)}\right)=\left\{T_{g} \mid\right.$ $g \in G\}$ and $C\left(T_{(0,1)}\right)=\left\{T_{(0, n)} \mid n \in \mathbb{Z}\right\}$.

(iii) $T_{(0,1)}$ and $T_{(0,2)}$ have the same family of factors. The map $K \mapsto \operatorname{Fix}(K)$ yields a one-to-one correspondence between the finite subgroups $K \subset G_{\infty}$ and the non-trivial factors of $T_{(0,1)}\left(\right.$ and $\left.T_{(0,2)}\right)$.

(iv) $T_{(0,1)}$ and $T_{(0,2)}$ have no prime factors.

(v) For any non-trivial factor $\mathfrak{F}$ of $T_{(0,1)}$, the restriction of $T_{(0,1)}$ to $\mathfrak{F}$ is near simple but not 2-fold simple and the restriction of $T_{(0,2)}$ to $\mathfrak{F}$ is simple.

(vi) The action of the subgroup $G_{\infty}$ is weakly mixing.

(vii) $T_{(0,1)}$ is disjoint from all simple transformations.

(viii) $T_{(0,1)}$ is disjoint from any distal extension of any simple transformation.

Proof. (i) It follows from Theorem 6.6 and Proposition 3.2 that $T_{(0,2)}$ is 2 -fold simple and $C\left(T_{(0,2)}\right)=\left\{T_{g} \mid g \in G\right\}$. By Proposition 6.7 and Corollary 1.2, $T_{(0,2)}$ is 3 -fold PID. Hence this transformation is 3 -fold simple (see Section 1). Therefore $T_{(0,2)}$ is simple by [GlHR].

(ii) Since $T_{(0,2)}$ is simple by (i), it follows from Proposition 3.2 that $T_{(0,1)}$ is near simple and $I\left(T_{(0,1)}\right)=I\left(T_{(0,2)}\right)=C\left(T_{(0,2)}\right)$. Since $T_{(0,2)}$ is simple, it is PID. Hence $T_{(0,1)}$ is PID by Corollary 1.2. It follows directly from Theorem 6.6 that $T_{(0,1)}$ is 2-fold QS (and hence QS) and non-2-fold simple.

(iii) follows from (i), (ii), Veech theorem ([Ve], [dJR]), Theorem 2.6 and the simple observation that every finite subgroup of $G$ is normal and is contained in $G_{\infty}$

(iv) follows immediately from (iii).

(v) We only need to show that $T_{(0,1)} \uparrow \mathfrak{F}$ is not 2 -fold simple since the other assertions of (v) follow from (i)-(iii), Proposition 3.4 and Corollary 3.5. If $\mathfrak{F}=$ $\operatorname{Fix}(K)$ then there exists $n>0$ such that $K \subset G_{n} \subset G_{\infty}$. Then for any $g \in G_{\infty} \backslash G_{n}$, the measure $0.5\left(\mu_{T_{g}}+\mu_{T_{g^{*}}}\right)\left\lceil\mathfrak{F} \otimes \mathfrak{F}\right.$ is an ergodic 2 -fold self-joining of $T_{(0,1)}\lceil\mathfrak{F}$. It is easy to see that this self-joining is 2 -to-one over its marginals. 
(vi) Let $\mathfrak{F}$ denote the sub- $\sigma$-algebra of subsets fixed by $\left(T_{g}\right)_{g \in G_{\infty}}$. Then $\mathfrak{F}$ is a factor of $T_{(0,1)}$. Now it follows from (iii) that $\mathfrak{F}$ is trivial. Hence the action of $G_{\infty}$ is ergodic. Since it commutes with the weakly mixing transformation $T_{(0,2)}$, this action is also weakly mixing.

(vii) Let $S$ be a simple transformation. If $S$ has pure point spectrum then it is disjoint with $T_{(0,1)}$ since the latter is weakly mixing. Hence it remains to consider the case where $S$ is weakly mixing. By (ii), $T_{(0,1)}$ is DS. Therefore if $T_{(0,1)}$ and $S$ are not disjoint then they have a non-trivial 'common' factor $\mathfrak{F}$ by Lemma 6.8. Since $\mathfrak{F}$ is a factor of $S$, it follows from Corollary 3.5 and Proposition 3.6 that either $S \uparrow \mathfrak{F}$ is simple or $S\lceil\mathfrak{F}$ is not 2 -fold near simple. However this contradicts to (v) because $S\left\lceil\mathfrak{F}\right.$ is isomorphic to $T_{(0,1)}\lceil\mathfrak{F}$.

(viii) follows from (vii) and $[\mathrm{Fu}]$.

\section{Some questions}

We list here several open problem related to near simple and DS actions.

(1) For which $l>1, l$-fold near simplicity of $G$ implies $(l+1)$-fold near simplicity? This question is especially interesting in particular cases when $G=\mathbb{R}$ and $G=\mathbb{Z}$.

(2) Whether any $l$-fold near simple action is $l$-fold DS?

(3) Whether the subsets of 2-fold QS and 2-fold DS transformations are meager in $\operatorname{Aut}_{0}(X, \mu)$ ?

I hope to consider these problems in a future paper.

\section{REFERENCES}

[Ag1] O. N. Ageev, The generic automorphism of a Lebesgue space conjugate to a G-extension for any finite abelian group G, Dokl. Akad. Nauk 374 (2000), 439-442. (Russian)

[Ag2] _ A typical dynamical system is not simple or semisimple, Erg. Th. \& Dynam. Syst. 23 (2003), 1625-1636.

[Da1] A. I. Danilenko, Comparison of cocycles of measured equivalence relations and lifting problems, Erg. Th. \& Dynam. Syst. 18 (1998), 125-151.

[Da2] _ Funny rank-one weak mixing for nonsingular Abelian actions, Isr. J. Math. 121 (2001), 29-54.

[Da3] Mixing rank-one actions for infinite sums of finite groups, Isr. J. Math. 156 (2006), 341-358.

[DaL] A. I. Danilenko and M. Lemańczyk, Isometric extensions, 2-cocycles and ergodicity of skew products, Studia Math. 137 (1999), 123-142.

[DaS] A. I. Danilenko and C. Silva, Mixing rank-one actions of locally compact Abelian groups, Ann. Inst. H. Poincaré, Probab. Statist. (to appear).

[dJ] A. del Junco, A simple map with no prime factors, Isr. J. Math. 104 (1998), 301-320.

[dJL1] A. del Junco and M. Lemańczyk, Simple systems are disjoint from Gaussian systems, Studia Math. 133 (1999), 249-256.

[dJL2] J Joinings of distally simple automorphisms, in preparation.

[dJR] A. del Junco and D. Rudolph, On ergodic actions whose self-joinings are graphs, Erg. Th. \& Dyn. Syst. 7 (1987), 531-557.

[Fel] J. M. G. Fell, A Hausdorff topology for the closed subsets of a locally compact nonHausdorff space, Proc. Amer. Math. Soc. 13 (1962), 472-476.

[Fe] S. Ferenczi, Systèmes de rang un gauche, Ann. Inst. H. Poincaré. Probab. Statist. 21 (1985), 177-186.

[FLe] K. Frączek and M. Lemańczyk, On mild mixing of special flows over irrational rotations under piecewise smooth maps, preprint.

[Fu] H. Furstenberg, Disjointness in ergodic theory, minimal sets and diophantine approximation, Math. Syst. Th. 1 (1967), 1-49. 
[Gl] E. Glasner, Ergodic theory via joinings, Mathematical Surveys and Monographs, 101, American Mathematical Society, Providence, RI, 2003.

[GlHR] E. Glasner, B. Host and D. Rudolph, Simple systems and their higher order self-joinings, Israel J. Math. 78 (1992), 131-142.

[GlK] E. Glasner and J. L. King, A zero-one law for dynamical properties, Topological dynamics and applications (Minneapolis, MN, 1995), 231-242, Contemp. Math., 215, Amer. Math. Soc., Providence, RI, 1998.

[GS] V. Ya. Golodets and S. D. Sinelshchikov, On the conjugacy and isomorphism problems for stabilizers of Lie group actions, Ergodic Theory \& Dynam. Systems 19 (1999), 391411.

[Ha] P. R. Halmos, Lectures on ergodic theory, Publications of the Mathematical Society of Japan, no. 3. The Mathematical Society of Japan, 1956.

[Ki1] J. L. King, Ergodic properties where order 4 implies infinite order, Israel J. Math. 80 (1992), 65-86.

[Ki2] ㄴ The generic transformation has roots of all orders, Colloq. Math. 84/85 (2000), $521-547$.

$[\mathrm{Ku}] \quad$ K. Kuratowski, Topology, Vol. I. New edition, revised and augmented. Academic Press, New York-London; Państwowe Wydawnictwo Naukowe, Warsaw, 1966.

[Li] E. Lindenstrauss, Pointwise theorems for amenable groups, Invent. Math. 146 (2001), 259-295.

[Ma] B. Madore, Rank-one group actions with simple mixing $\mathbb{Z}$-subactions, New York J. Math. 10 (2004), 175-194.

[Or] D. S. Ornstein, On the root problem in ergodic theory, Proc. Sixth Berkley Symp. Math. Stat. Prob. (Univ. California, Berkeley, Calif., 1970/1971), vol. II, Univ. of California Press, Berkeley, Calif., 1972, pp. 347-356.

[Par] K. K. Park, GL(2, Z) action on a two torus, Proc. Amer. Math. Soc. 114 (1992), 955963.

[Pa] K. R. Parthasarathy, A note on idempotent measures in topological groups, J. London Math. Soc. 42 (1967), 534-537.

[PRy] A. A. Prikhod'ko and V. V. Ryzhikov, Simple noncommutative counterexamples in joining theory, Vestnik Moskov. Univ. Ser. I Mat. Mekh. (1998), 16-19 (Russian); translation in Moscow Univ. Math. Bull. 53 (1998), 15-17.

[Ra] A. Ramsay, Virtual groups and group actions, Adv. in Math. 6 (1971), 253-322.

[Rat] M. Ratner, Horocycle flows, joinings and rigidity of products, Ann. of Math. (2) 118 (1983), 277-313.

[Ru] D. J. Rudolph, An example of a measure preserving map with minimal self-joinings, J. d'Analyse Math. 35 (1979), 97-122.

[Ry1] V. V. Ryzhikov, Even and odd simplicity of dynamical systems with an invariant measure, Mat. Zametki 60 (1996), 470-473 (Russian); translation in Math. Notes 60 (1996), $353-356$.

[Ry2] V. V. Ryzhikov, Around simple dynamical systems. Induced joinings and multiple mixing, J. Dynam. Control Systems 3 (1997), 111-127.

[RyT] V. V. Ryzhikov and J.-P. Thouvenot, Disjointness, divisibility and quasi-simplicity of measure-preserving actions, Funktsional. Anal. i Prilozhen. 40 (2006) (to appear).

[So] A. Sokhet, Les actions approximativement transitives dans la théory ergodique, Thèse de doctorat, Université Paris VII, 1997.

[Th1] J.-P. Thouvenot, Some properties and applications of joinings in ergodic theory, Ergodic theory and its connections with harmonic analysis (Alexandria, 1993), 207-235, London Math. Soc. Lecture Note Ser., 205, Cambridge Univ. Press, Cambridge, 1995.

[Th2] Les systemes simples sont disjoints de ceux qui sont infiniment divisibles et plongeables dans un flot, Colloq. Math. 84/85 (2000), 481-483.

[Va] V. S. Varadarajan, Groups of automorphisms of Borel spaces, Trans. Amer. Math. Soc. 109 (1963), 191-220.

[Ve] W. A. Veech, A criterion for a process to be prime, Monatsh. Math. 94 (1982), 335-341.

[Zi] R. J. Zimmer, Extensions of ergodic group actions, Illinois J. Math. 20 (1976), 373-409.

Institute for Low Temperature Physics \& Engineering of Ukrainian National Academy of Sciences, 47 Lenin Ave., Kharkov, 61164, UKRAine

E-mail address: danilenko@ilt.kharkov.ua 